\title{
URLLC and eMBB in 5G Industrial loT: A Survey
}

This paper was downloaded from TechRxiv (https://www.techrxiv.org).

\section{LICENSE}

CC BY 4.0

SUBMISSION DATE / POSTED DATE

02-02-2022 / 03-02-2022

\section{CITATION}

Sharfeen, Benish; Jangsher, Sobia; ahmed, ashfaq; Al-Dweik, Arafat (2022): URLLC and eMBB in 5G Industrial loT: A Survey. TechRxiv. Preprint. https://doi.org/10.36227/techrxiv.19106795.v1

$\mathrm{DOI}$ 


\title{
URLLC and eMBB in 5G Industrial IoT: A Survey
}

\author{
Benish Sharfeen Khan, Sobia Jangsher, Member, IEEE, \\ Ashfaq Ahmed, Senior Member, IEEE, Arafat Al-Dweik, Senior Member, IEEE
}

\begin{abstract}
Fifth generation (5G)-industrial Internet of things (IIoT) is the integration of IIoT and a private $5 \mathrm{G}$ network. The IIoT is a concept that involves incorporating smart objects, gadgets, and solutions into cutting-edge industrial operations to increase reliability, efficiency, and over-production costs. Furthermore, the integration of IIoT and 5G/beyond 5G (B5G) provides the potential for ubiquitous and instantaneous connectivity. The 5G architecture can handle the IIoT's stringent ultra-low latency, real-time processing, high data-rate, nearby storage, and reliability requirements. A new era of economic growth is predicted for IIoT assisted 5G/B5G wireless networks. It should be noted that the majority of the work in IIoT is focused on the architecture, with reliability and throughput being largely ignored. This paper provides a comprehensive review of B5G assisted IIoT wireless networks, with a focus on enhanced mobile broadband (eMBB) and ultra-reliable low latency communication (URLLC) services. Furthermore, it provides insights into various applications and key enabling technologies from the perspective of URLLC, eMBB and their tradeoff.
\end{abstract}

Index Terms-fifth generation (5G), beyond 5G (B5G), sixth generation (6G), industrial Internet of things (IIoT), ultrareliable low latency communication (URLLC), enhanced mobile broadband (eMBB).

\section{INTRODUCTION}

$\mathbf{T}$ $\mathrm{HE}$ emergence of industrial Internet of things (IIoT) has revolutionized industrial operations such as manufacturing and production by automating a huge number of connected components and devices. IIoT is a sub-category of Internet of things (IoT) that focuses on the use of IoT techniques and technologies [1]-[4] in industries such as smart cities, smart transportation, smart grid, smart health services, forestry, food, weather, agriculture, monitoring, and surveillance [5][18]. The machines/devices in IIoT are connected to capture smartness and autonomy in legacy systems [19]-[21]. It is estimated that by the end of 2030, roughly 80 billion devices will be connected to the Internet [22]-[24]. In IIoT, an increased degree of connectivity is involved, which has special requirements for high reliability, low latency, high speed, flexible, and secure communication [25]-[27].

Benish Sharfeen Khan is with the Department of Electrical Engineering, Institute of Space Technology, Islamabad, Pakistan. (email: beenish09@ist.edu.pk).

Sobia Jangsher is with the Department of Electrical Engineering and Computer Science, Khalifa University, 127788 Abu Dhabi, UAE, and also with the Department of Electrical Engineering, Institute of Space Technology, Islamabad, Pakistan. (email: sobia.jangsher@ku.ac.ae; sobia.jangsher@ist.edu.pk).

Ashfaq Ahmed is with the Department of Electrical Engineering and Computer Science, Khalifa University, 127788 Abu Dhabi, UAE (email: ashfaq.ahmed@ku.ac.ae).

A. Al-Dweik is with the Center for Cyber-Physical Systems (C2PS), Khalifa University, 127788 Abu Dhabi,UAE, and also with the Department of Electrical and Computer Engineering, Western University, London,ON N6A 3K7, Canada (e-mail: dweik@fulbrightmail.org; arafat.dweik@ku.ac.ae);
Forth generation $(4 \mathrm{G})$ wireless networks has gained popularity over the past decade as a reliable communications technology [27]-[29]. Many industrial applications are supported by long term evolution (LTE) and wireless fidelity (WiFi), but with more demanding performance needs from users in terms of reliability, latency, and throughput, fifth generation (5G) has emerged as a promising alternative that can satisfy these requirements [30]. 5G started becoming commercially available in 2019. It is used a wide range of applications from IoT to smart home to Industry(4.0). Moreover, the International Telecommunication Union (ITU) defines three usage scenarios: ultra-reliable low latency communication (URLLC), enhanced mobile broadband (eMBB), and massive machine type communication (MTC) (mMTC) [31]-[34]. To sustain/ achieve the competitive requirements for the applications of wireless networks, the industry and academia have started to conceptualize the next generation of wireless communication systems (sixth generation (6G)) [35]-[37]. The 6G is expected to have a data rate of $20 \mathrm{Gbps}$, admit $10^{6}$ devices per $\mathrm{km}^{2}$, and have a latency/delay of less than $1 \mathrm{~ms}$. Thus, 5G/6G enhancements are capable of providing the essential services and meeting the performance metrics of IIoT.

For integration of $5 \mathrm{G} / 6 \mathrm{G}$ with IIoT, a private $5 \mathrm{G}$ network can be designed and deployed [38]-[40]. The requirement for a private network has been addressed in the $5 \mathrm{G}$ standards, rather than being an add-on capability in previous generations. The primary motive for establishing a private $5 \mathrm{G}$ network is the guaranteed coverage as well as the better performance profile over legacy wireless technologies. Because most industries are located in distant places where public network coverage is limited or non-existent, a private network can provide the assured service required by IIoT. It is a secure network customized for a certain industry, with a dedicated radio access network (RAN) and core mobile network for mobile communication. As a result, a private $5 \mathrm{G}$ network for IIoT known as 5G-IIoT could be a promising solution.

5G-IIoT can be used to complement the existing communication solutions for IIoT and can be used based on application requirements. The two most characteristic features of 5G-IIoT are a) URLLC, which requires a latency of less than $1 \mathrm{~ms}$ and a reliability of $99.99 \%$, and b) eMBB, which requires a data rate of giga bits per second. Therefore, 5G-IIoT may achieve extremely high data-rates, low latency with wide coverage, and relatively low power consumption [41], [42].

\section{A. Existing Surveys}

Table I provides a summary of the existing surveys on the IIoT. Xu et al. [43] outlines a comprehensive survey work for IIoT. This work covers current research in IoT technology, 
TABLE I: An overview of existing surveys on IIoT. Rel.: Reliability, Tp.: Throughput, Appl.: Applications, Chall.: Challenges.

\begin{tabular}{|c|c|c|c|c|c|c|c|c|}
\hline Ref. & Title & $5 G / 6 G$ & Techniques & $\begin{array}{c}\text { Rel. } \\
\text { (URLLC) }\end{array}$ & $\begin{array}{c}\text { Tp. } \\
\text { (eMBB) }\end{array}$ & Trade-off & Appl. & Chall. \\
\hline [43] & IoT in industries: A survey & & $\bar{\nabla} \checkmark$ & & & & $\bar{\nabla}$ & $\overline{\mid l \checkmark}$ \\
\hline [44] & $\begin{array}{l}\text { IIoT: Challenges, opportunities, } \\
\text { and directions }\end{array}$ & & & & & & & $\checkmark$ \\
\hline [19] & $\begin{array}{l}\text { Industrial IoT in } 5 \mathrm{G} \text { environment } \\
\text { towards smart manufacturing }\end{array}$ & $\checkmark$ & $\checkmark$ & & & & & $\checkmark$ \\
\hline$[45]$ & $\begin{array}{c}\text { Communication protocols of an } \\
\text { IIoT environment: A comparative } \\
\text { study }\end{array}$ & & $\checkmark$ & & & & & \\
\hline$[46]$ & $\begin{array}{l}\text { Creating values out of IoT: An } \\
\text { industrial perspective }\end{array}$ & & & & & & & $\checkmark$ \\
\hline [47] & $\begin{array}{l}\text { 5G URLLC implementation } \\
\text { challenges and operational issues } \\
\text { with IoT devices }\end{array}$ & $\checkmark$ & & $\checkmark$ & $\checkmark$ & & $\checkmark$ & \\
\hline$[20]$ & $\begin{array}{l}\text { URLLC wireless communication: } \\
\text { Tail, risk and scale }\end{array}$ & & & $\checkmark$ & & $\checkmark$ & $\checkmark$ & \\
\hline$[21]$ & $\begin{array}{l}\text { A comprehensive survey on IoT } \\
\text { towards } 5 \mathrm{G} \text { wireless systems }\end{array}$ & $\checkmark$ & $\checkmark$ & & & & $\checkmark$ & $\checkmark$ \\
\hline$[48]$ & $\begin{array}{l}\text { A Energy-Efficient IIoT: } \\
\text { Overview and Open Issues }\end{array}$ & & $\checkmark$ & & & & & $\checkmark$ \\
\hline $\begin{array}{l}\text { This } \\
\text { work }\end{array}$ & $\begin{array}{l}\text { URLLC and eMBB Support for } \\
\text { IIoT: Techniques, Challenges, } \\
\text { Applications and Research Gaps }\end{array}$ & $\checkmark$ & $\checkmark$ & $\checkmark$ & $\checkmark$ & $\checkmark$ & $\checkmark$ & $\checkmark$ \\
\hline
\end{tabular}

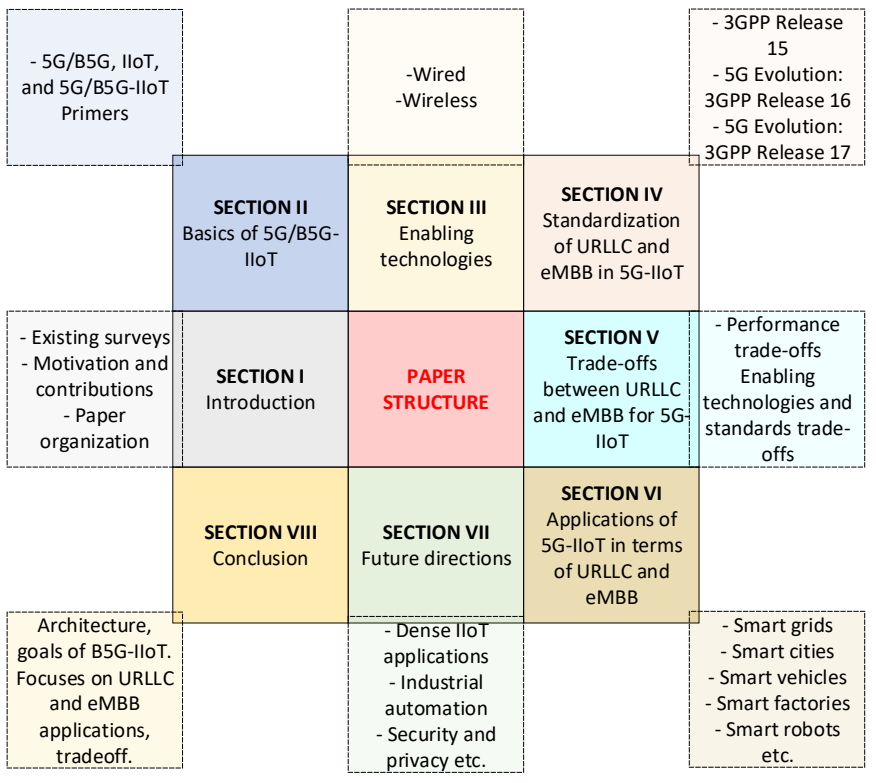

Fig. 1: Structure of paper.

applications, and industry issues. In [44], a brief introduction toIoT, IIoT, and industry 4.0, with emphasis on performance, parameters, research efforts, and future directions in the domain is provided. The implementation methods, manufacturing technologies, and scenarios of beyond 5G (B5G)-IIoT based cyber-physical manufacturing systems (CPMS) are discussed in detail in [19]. Event based polling protocols for IIoT are briefly discussed in [45]. Various applications in the domains ofIoT cloud platform with industrial perspective are debated in [46]. Further, the challenges of cloud based IoT are inves- tigated to generate potentially more business opportunities in industries. In [47], the progress in third generation partnership project (3GPP), and implementation issues of URLLC in IoT devices is briefly addressed. In [20], the main focus is on requirements, techniques and methodologies related to URLLC to ensure the goals of $5 \mathrm{G}$ wireless networks. A comprehensive survey of IoT in 5G system is provided in [21]. It provides a review of enabling and emerging technologies of $5 \mathrm{G}$ in IoT with research gaps. Finally, in [48], challenges and technologies in perspective of energy efficiency are discussed for IIoT.

\section{B. Motivation and Contributions}

Although there are current surveys on IIoT, based on extensive literature search and to the best of the authors knowledge, none of them focus on the IIoT reliability and throughput aspects. URLLC and IIoT have a lot of potential and a promising future in 5G-IIoT. However there is a trade-off between both technologies, which motivated us to investigate IIoT features and requirements, as well as its enabling techniques. We also cover the trade-offs and challenges encountered by IIoT in URLLC and $\mathrm{eMBB}$, as well as future directions.

The following summarizes the major contributions of this work:

- Presents a comprehensive literature review for IIoT in 5G to determine how $5 \mathrm{G}$ fits the requirements of IIoT. The prime objective of this work is to identify research gaps in the URLLC and eMBB aspects of IIoT in 5G.

- Provides insights into diverse applications and requirements, where we investigate the key enabling technologies for 5G-IIoT, particularly the communication technologies for URLLC and eMBB. 


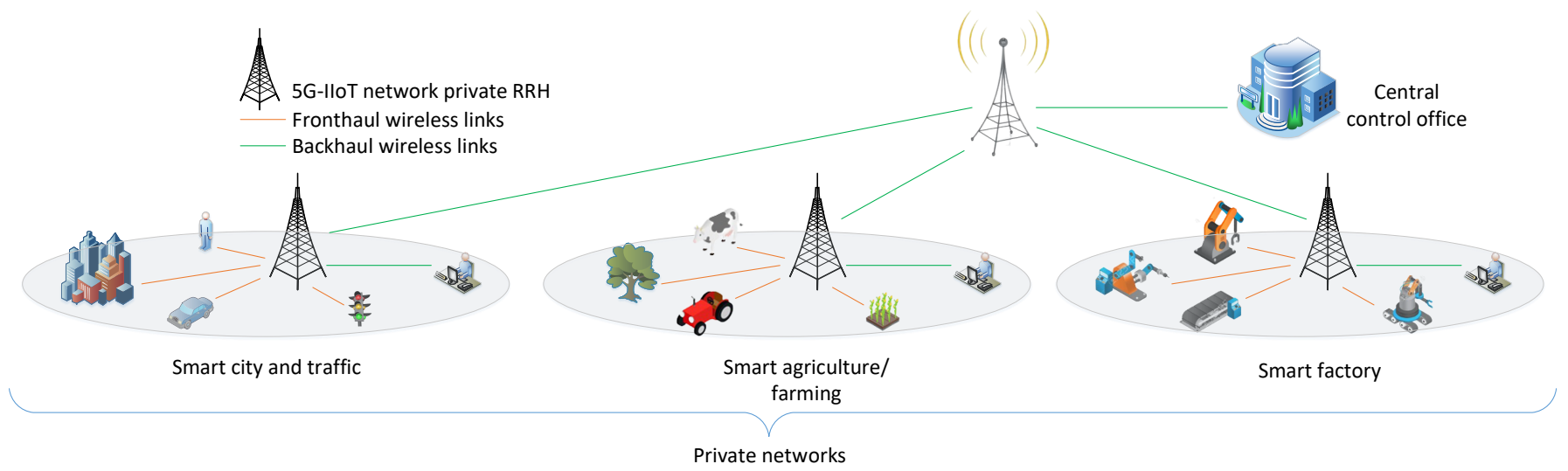

Fig. 2: 5G-IIoT private network.

- Examines the trade-off between URLLC and eMBB.

- Outlines the challenges and future directions for 5G-IIoT for URLLC and eMBB.

\section{Paper Organization}

The organization of the paper is as follows: The primer of B5G and IIoT and their relationship is briefly discussed in section II. The enabling techniques for URLLC and eMBB, and their role in IIoT is provided in Section III. Section IV presents $5 \mathrm{G}$ standardization for IIoT with respect to URLLC and eMBB. Section V describes the trade-off between URLLC and eMBB. The applications of B5G-IIoT are discussed in Section VI. The challenges and future directions are debated in Section VII. The paper is summarized in Section VIII. The overall structure of the paper is shown in Fig. 1.

\section{BASICS OF 5G/B5G-IIOT}

The core architecture of 5G/B5G and IIoT is covered in this section, as well as the 5G-IIoT design and objectives.

\section{A. 5G/B5G Primer}

The ecosystem of $5 \mathrm{G} / \mathrm{B} 5 \mathrm{G}$ connects the entire society through digital devices and gadgets, driving the economic development of innovative services. Aside from network virtualization, the flexible and dynamic architecture of 5G/B5G offers numerous access technologies with unlicensed and licensed users and devices. The 5G/B5G network may be scaled and extended in cost-effective and automated models to support revolutions in the automotive, manufacturing, utilities, transportation, public safety, healthcare, media, and other industries. The 5G/B5G architecture supports a wide range of advanced access technologies, including $\mathrm{WiFi}$, B5G, new radio (NR), low power wide area (LPWA), and cellular with interaccess mobility [26], [49], [50]. The main features are built on software-defined-networking (SDN) and cloud/virtualization native principles, with full orchestration to provide flexible deployments.

Potential use cases for B5G with varying requirements have been identified by standard bodies and industrial parties. These prospective use cases can be divided into three categories:
- eMBB: can be used to service large-scale events and compact metropolitan areas that have high data rate requirements but restricted bandwidth. Virtual and augmented reality, smart offices, $8 \mathrm{~K} / 4 \mathrm{~K}$ video streaming, and cloud applications all require broadband connectivity with a minimum data rate of $50 \mathrm{Mbps}$ everywhere. Furthermore, mobile enabled eMBB services are necessary for enhanced navigation, in-vehicle infotainment, telematics support for diagnostics and safety, and commercial aircraft on-board entertainment.

- URLLC: Mission-critical communication is required for emergency services such as disaster response, public safety, and location services. URLLC provides real-time services for scenarios requiring a response time of less than $1 \mathrm{~ms}$. Among the use cases covered by URLLC are robotic control-based industrial automation, autonomous driving, remote surgery, and drones [51].

- Massive IoT (mIoT): Long-range, low-cost, and ultraenergy-sensitive devices that require infrequent and periodic connectivity from cloud applications and remote locations are examples of mIoT. LPWA cellular technologies were provided in 3GPP release-13 with long term evolution for machine (LTE-M) and narrowband Internet of things (NB-IoT), and further advances were proposed in release-14, which are aligned with the improvements of the $5 \mathrm{G} / \mathrm{B} 5 \mathrm{G}$ architecture.

\section{B. IIoT Primer}

The emergence of digital and smart manufacturing in industries aims to merge operational technology (OT) and information technology (IT). In general, the IIoT will connect industrial assets, such as machinery and control systems, to the IT and business processes. This integration results in massive data generation and collection. This data could be used to develop analytic solutions to improve industrial operations [52]. Smart manufacturing, on the other hand, concentrates primarily on the production stage of a smart product's lifecycle, with the aim of responding quickly and dynamically to demand changes. As a result, IIoT has an impact on the industrial value chain and meets the standards for smart manufacturing. In general, the machines in IIoT are meant 
to communicate autonomously with one another. The IIoT comprises situations such as legacy monitoring applications, i.e., process monitoring in manufacturing plants, and novel ways for self-organizing systems, i.e., autonomic industrial plants with minimal human intervention.

Typically, IIoT systems are envisioned as a layered modular architecture of digital technologies. The physical components, such as cyber-physical systems (CPS), sensors, or machines, are referred to as the device layer. The network layer consists of physical network buses, cloud computing, and communication protocols that collect and transfer data to the service layer, which consists of applications that transform and combine data to display information on the driver dashboard. The content layer, also known as the user interface, is the topmost layer of the stack. The user interface devices, such as computer screens, point of sale (PoS) stations, tablets, smart glasses, and smart surfaces, are included in this layer. The service layer follows, which includes applications and software for analyzing collected data and transforming it into usable information. It then proceeds to the network layer, where several communication protocols such as $\mathrm{WiFi}$, Bluetooth, and low range (LoRa) operate. The device layer contains the physical hardware of the IIoT network, including devices such as CPS, machines, and sensors.

Standard bodies and industry stakeholders have identified a variety of potential use cases for IIoT. The following are the general requirements and objectives of IIoT:

- Quality of service (QoS) requirement with low latency, and ultra high reliability is vital.

- Low cost scalable network with esteemed security and privacy is desired.

- The emerging standards should be smoothly implemented and integrated on the IIoT devices in a flexible way.

- Inter- and Intra-connection of networks and IoT devices should be possible frequently.

\section{Does 5G/B5G Satisfy IIoT Requirements?}

After understanding the fundamental and necessary needs of IIoT, it is critical to establish if present and future wireless communication standards meet these requirements. The current standard, $5 \mathrm{G}$, provides use cases that make industrial systems more flexible, beneficial, and autonomous while meeting QoS requirements of both 5G/B5G and IIoT. Some of the goals that can be reached with $\mathrm{B} 5 \mathrm{G}$ in IIoT are as follows:

- Optimized services can be provided through the use of a dedicated network and unified connectivity.

- The standard guarantees $99.999 \%$ reliability with less than $1 \mathrm{~ms}$ latency in radio frequency (RF) environments.

- It substitutes fixed wired Ethernet lines with reconfigurable communication technology.

- Private networks can operate on both licensed and unlicensed spectrum.

\section{5G/B5G-IIoT Primer}

The system model in Fig. 2 depicts the high layer connectivity found in a 5G-IIoT network. 5G/B5G-IIoT is an integration

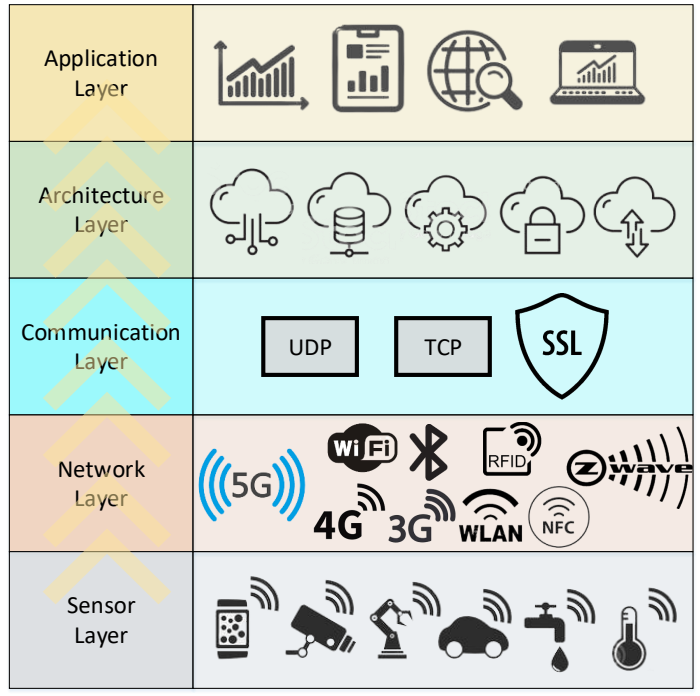

Fig. 3: 5G-IIoT architecture.

of $5 \mathrm{G} / \mathrm{B} 5 \mathrm{G}$ with IIoT over a private $5 \mathrm{G}$ network. The inclusion of $5 \mathrm{G} / \mathrm{B} 5 \mathrm{G}$ in IIoT can provide the required ubiquitous and instantaneous connectivity for IIoT applications. A private network is one that has its own dedicated RAN and core mobile network. It requires a framework to process and analyze the obtained data using inter- and intra-industrial/device units. As shown in Fig. 3, the B5G-IIoT architecture is primarily separated into five levels [26], [27], [50], [53].

- Application Layer This layer includes IIoT applications in B5G, such as smart grid, smart factory, smart city, smart cars, traffic management system, health, and education. Devices and machines that send data via the internet are also included in the application layer.

- Architecture Layer This layer incorporates big data analytic (BDA) cloud computing and edge computing for data processing.

- Communication Layer The communication layer of the IIoT network transports information between all layers and is considered a critical component of the network.

- Network Layer This layer includes communication technologies such as low power wide area network (LPWAN), Bluetooth, WiFi, and 802.11.x, depending on the range and various required factors.

- Sensor Layer In the physical layer, sensors and actuators are available. These sensors are in charge of collecting data and transmitting it to the upper layer, i.e., the network layer.

The modern industrial industry is undergoing a technological transformation. Meanwhile, the IIoT proposes new communication technology requirements. Every IIoT application has essential design goals to improve QoS requirements. The key design goals for these critical IIoT applications are depicted in Fig. 4. The key design objectives are briefly stated here.

- Energy: Typically, IoT devices are classified as low power devicess (LPDs), with limited on-board energy sources. As a result, in order to improve the performance of the IIoT network, these LPDs should make efficient use 

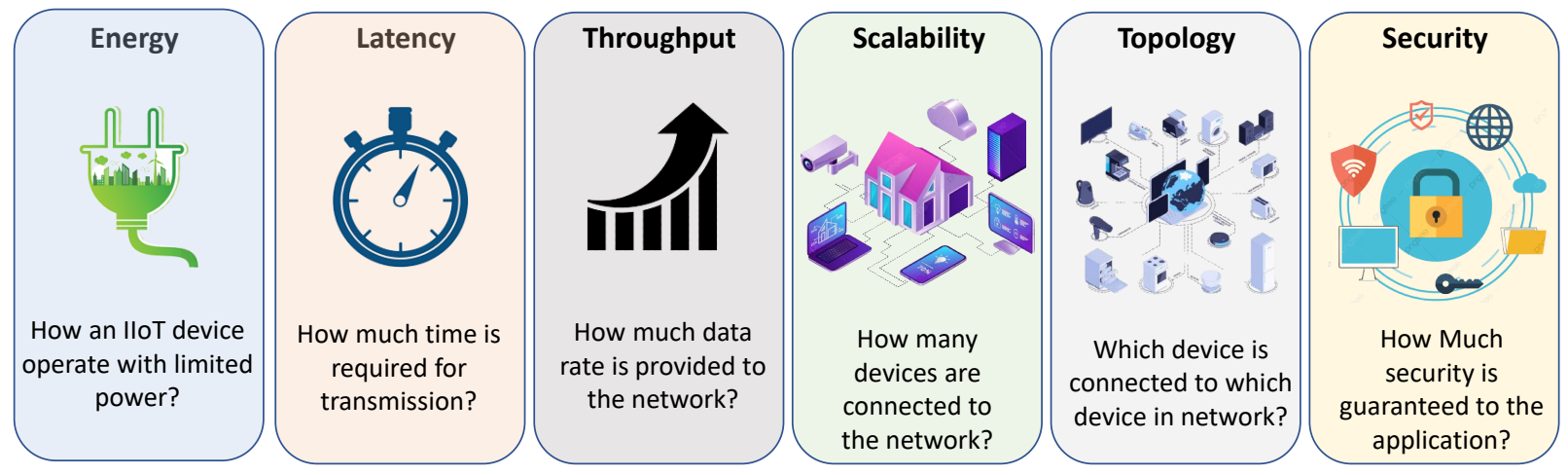

Fig. 4: Design goals for 5G-IIoT.

of their available power resources to extend their life.

- Latency: In general, the B5G-IIoT applications are timesensitive. As a result, a minimum end-to-end latency of less than $1 \mathrm{~ms}$ must be reached in all sorts of processing, computation, and propagation in the B5G-IIoT applications.

- Throughput and coverage: The QoS requirements vary for different B5G-IIoT applications and can be fulfilled by utilizing several efficient protocols. Typically, B5GIIoT applications require a throughput in Gbps. For efficient transmission, long-range IIoT devices with longlife batteries must be used.

- Network topology: The number of devices connected to other devices, as well as to cloud and fog architectures, must be carefully determined for improved QoS. Furthermore, network topology has a significant impact on QoS performance.

- Security \& safety: Nowadays, security and privacy are key concerns. To avoid the leakage of confidential information or data loss, data collection and processing between inter- and intra-B5G-IIoT applications require strong privacy and security.

- Reliability: Many B5G-IIoT applications require ultrahigh reliability. For example, in a smart healthcare system, it is critical to deliver data reliably. Furthermore, the QoS requirements of URLLC in B5G-IIoT demand high reliability in a variety of real-time applications.

- Cost effective: Low-cost B5G-IIoT smart devices and applications must be installed and used to reduce capital expenditure (CAPEX)/operating expenditures (OPEX). Deployment of applications such as smart factories and industries must satisfy market demands.

- Standardization: In IIoT, the many B5G standards are employed. These standards' implementation must be flexible and compatible with IIoT devices.

- Device maintenance: In an industry 4.0 environment, heterogeneous devices require constant management due to their connectivity to one another and to the Internet. SDN is used to describe such failures and the evolution of IIoT device maintenance issues.

- Monitoring network: Mobility, congestion, and overload of IIoT devices within a wireless network can all affect the network topology. As a result, continual and frequent monitoring of the system is unavoidable. The number of smart devices in a IIoT application will grow over time. Thus, to avoid traffic congestion and overload, the system's settings should be capable of being adjusted in response to the traffic load and data requirements.

- Configuration \& system management: It is critical to empower devices with self-configuration and control capabilities, as well as to reconfigure the network to accommodate new devices.

- Scalability and integration: Scalability poses various problems, such as how many smart devices are required to support an industrial application environment, how many devices can be comfortably handled by a server, and how to design a system efficiently within energy/spectrum constraints. Additionally, the integration of hybrid SDN and IIoT devices must be seamless and optimal.

- Heterogeneity \& interoperability: Heterogeneous intelligent IIoT devices must communicate and exchange data with other devices, as well as share it through the Internet. This integration raises some serious challenges that must be addressed. Furthermore, standardization is desired for IIoT device interoperability.

\section{ENABLING AND Existing TeChNiQues FOR 5G-IIoT}

IIoT networks have a wide range of applications in electronic devices and industrial equipment, and are connected to billions of people. These numerous devices support a variety of network protocols and communication technologies; additionally, they are equipped with a variety of data processing and storage units and operate at a range of power levels. Each device is constrained by QoSs, energy efficiencys (EEs), and spectral efficiencys (SEs)/throughput, cost, power, security, reliability, and latency/delay. Industrial communication technologies are classified into wired and wireless categories according to their transmission procedures, and account for $85 \%$ of the global market [54]-[57]. IIoT communication technologies are illustrated in Fig. 5.

\section{A. Wired Communication Technologies}

Wired technologies include industrial field-bus and industrial Ethernet. These technologies provide reliable communication between the floor's control system and the higherlevel control system. For cross-region communication between 

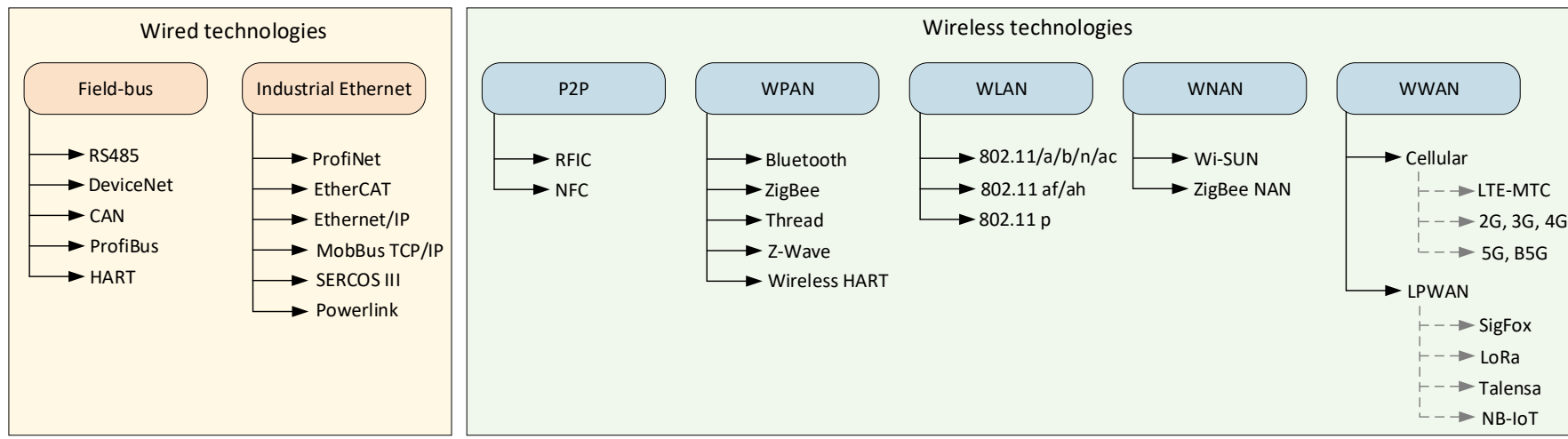

Fig. 5: IIoT communication technologies.

floors, control systems, and data centers, traditional Ethernet with its complex line topology is used, resulting in significant delays and inadequate security.

1) Field-bus: Field-bus communication has been used to connect actuators, sensors, and instruments. Moreover, data was transferred through field bus between these devices and higher control systems. It is the most extensively used wired industrial communication technology, consisting of RS485, DeviceNet, controller area network (CAN), process field bus (ProfiBus), and highway addressable remote transducer (HART).

2) Industrial Ethernet: For IIoT, industrial Ethernet is a suitable wired technology. When a huge amount of data needs to be conveyed, it gives a greater bandwidth than field bus technology. Ethernet protocols are becoming more prevalent as control system technologies evolve. The manufacturing execution system (MES) and supervisory control and data acquisition (SCADA) are examples of these control systems. Industrial Ethernet protocols include process field net (ProfiNet), ethernet for control automation technology (EtherCAT), Ethernet/internet protocol (IP), modbus transmission control protocol (TCP)/IP, serial real-time communication system (SERCOS)-III, and Power-Links.

\section{B. Wireless Communication Technologies}

There are numerous wireless technologies that can be used in IIoT. These technologies are classified into long and short range technologies based on their coverage range. Fig. 5 illustrates the major wireless technologies. The technologies are further categorised according to the services they offer, the requirements they fulfill, and the characteristics they possess [41], [58]-[63]. Table II summarizes the major parameters of the existing wireless technologies employed in IIoT. These technologies are described briefly in the following section.

1) Peer to peer $(P 2 P)$ : $\mathrm{P} 2 \mathrm{P}$ technology promotes the implementation of a cost-effective real-time infrastructure for IIoT that is both secure and low-latency [64], [65].

- Radio-frequency identification (RFID) is a wireless technology that is well suited for real-time, short-range, low-power, and low-cost communication in IIoT. It is used to transport small volumes of data between servers and devices in a short period of time.
- Near field communication (NFC) is a contactless data exchange high frequency RFID technology with low power consumption that is used to accomplish the shortest range possible. NFC is used to authenticate cards and facilitates server-to-server communication via P2P.

2) Wireless personal area network (WPAN): WPAN has a range of up to 10 to 100 meters and is used to connect lowpower devices that are centrally located [66], [67]. WPAN is a collection of low-cost devices such as bluetooth, zonal intercommunication global-standard (ZigBEE), Thread, Z-wave, and Wireless Hart.

- Bluetooth is classified as WPAN and is widely regarded as the most appropriate technology for IIoT applications requiring low power. Initially, it supported only P2P communication, but was later expanded to support multi-point communication between up to 7 nodes for consumers.

- ZigBEE is also referred to as IEEE 802.15.4, is a lowpower, short-range WPAN technology. It is optimal for medium-range IIoT applications that require a higher data rate. Home automation and sensor networks are two examples of IIoT applications.

- Thread is WPAN technology which is based on internet protocol version 6 (IPV6) low power mesh networking protocol. It is well suited for IIoT applications that emphasize functional efficiency, automation, and security. It is designed with the challenging features of low power consumption, a simple architecture, and enhanced security with no single point of failure. It is used for device-to-device and device-to-cloud communication and supports up to 250 nodes.

- Z-Wave is categorized as WPAN and is primarily used for IIoT home control and automation applications. Zwave is a low-cost short-range network with a $200 \mathrm{~ms}$ latency. It is capable of supporting up to 232 devices via a mesh network and operates flawlessly without any coordination. However, it performs optimally with $30-50$ nodes, according to the manufacturer.

- Wireless HART communication is a two-way WPAN technology. It is designed to meet self-organizing and self-healing requirements using existing HART technologies. These are the requirements for flexibility, reliability, and security. It is optimal for IIoT applications that 
TABLE II: Wirless technologies used in IIoT.

\begin{tabular}{|c|c|c|c|c|c|c|c|c|}
\hline \multicolumn{3}{|c|}{ Technology } & Range (m) & Frequency & Max. Bandwidth & Data Rate & Power & Cost \\
\hline $\mathrm{P} 2 \mathrm{P}$ & \multicolumn{2}{|c|}{ RFID } & $10 \mathrm{~m}$ & $13.56 \mathrm{MHz}$ & $1 \mathrm{MHz}$ & $640 \mathrm{Kbps}$ & Low & Low \\
\hline \multirow{4}{*}{ WPAN } & \multicolumn{2}{|c|}{ Bluetooth } & $60 \mathrm{~m}$ & $2.402-2.480 \mathrm{GHz}$ & $2 \mathrm{MHz}$ & $1 \mathrm{Mbps}$ & Medium & \multirow{3}{*}{ Low } \\
\hline & \multirow{2}{*}{\multicolumn{2}{|c|}{ Thread }} & $140 \mathrm{~m}$ & ISM $<2.4 \mathrm{GHz}$ & $5 \mathrm{MHz}$ & $40 \mathrm{Kbps}, 250 \mathrm{Kbps}$ & \multirow{3}{*}{ Low } & \\
\hline & & Nave & $100 \mathrm{~m}$ & $868.42 \mathrm{MHz}-908.42 \mathrm{MHz}$ & $200 \mathrm{KHz}$ & 9.8-100 Kbps & & \\
\hline & \multicolumn{2}{|c|}{ Wireless-Hart } & $225 \mathrm{~m}$ & $2405-2480 \mathrm{GHz}$ & $5 \mathrm{MHz}$ & $250 \mathrm{Kbps}$ & & High \\
\hline \multirow{5}{*}{ WLAN } & \multirow{5}{*}{802.11} & $\mathrm{~b}$ & $\begin{array}{l}\text { Indoor } 35 \mathrm{~m} \text {, } \\
\text { Outdoor } 140 \mathrm{~m}\end{array}$ & $2.4 \mathrm{GHz}$ & $20 \mathrm{MHz}$ & 1-11 Mbps & \multirow{5}{*}{ Low } & \multirow{5}{*}{ Low } \\
\hline & & $\mathrm{n}$ & $\begin{array}{c}\text { Indoor } 70 \mathrm{~m} \text {, } \\
\text { Outdoor } 250 \mathrm{~m}\end{array}$ & $2.4-5 \mathrm{GHz}$ & $20-40 \mathrm{MHz}$ & 72-600 Mbps & & \\
\hline & & $\mathrm{ac}$ & $100-1000 \mathrm{~m}$ & $5 \mathrm{GHz}$ & $20,40,60,80 \mathrm{MHz}$ & 433-6933 Mbps & & \\
\hline & & af & \multirow[b]{2}{*}{$1000 \mathrm{~m}$} & $0.054-0.799 \mathrm{GHz}$ & $6-8 \mathrm{MHz}$ & $<568.9 \mathrm{Mbps}$ & & \\
\hline & & ah & & Sub GHz & $1-16 \mathrm{MHz}$ & $150 \mathrm{kbps}-78 \mathrm{Mbps}$ & & \\
\hline \multirow{7}{*}{ WWAN } & \multirow{4}{*}{ LPWAN } & SigFox & $\begin{array}{l}30 \text { miles rural area, } \\
2-6 \text { miles urban area }\end{array}$ & $\begin{array}{c}868 \mathrm{MHz} \text { Europe, } 915 \\
\text { MHz North America, } 433 \\
\text { MHz Asia }\end{array}$ & $100 \mathrm{~Hz}$ & $100 \mathrm{bps}$ & Low & \multirow{4}{*}{ Low } \\
\hline & & LoRa & $15-20 \mathrm{~km}$ & $\begin{array}{c}868 \mathrm{MHz} \text { Europe, } 915 \\
\text { MHz North America, } 433 \\
\text { MHz Asia }\end{array}$ & $250 \mathrm{KHz}$ and $125 \mathrm{KHz}$ & $50 \mathrm{Kbps}$ & $\begin{array}{c}\text { Device } \\
\text { dependent }\end{array}$ & \\
\hline & & Telensa & $\begin{array}{c}2 \mathrm{~km} \text { urban area, } 4 \\
\mathrm{~km} \text { rural area }\end{array}$ & Sub GHz & - & $62.5-500 \mathrm{bps}$ & \multirow[t]{2}{*}{ Low } & \\
\hline & & NB-IoT & $\begin{array}{c}1 \mathrm{~km} \text { urban area, } 10 \\
\mathrm{~km} \text { rural area }\end{array}$ & Licensed LTE & $200 \mathrm{KHz}$ & $200 \mathrm{Kbps}$ & & \\
\hline & \multirow{3}{*}{ Cellular } & LTE-MTC & $<100 \mathrm{~km}$ & 4G-LTE Band & $20 \mathrm{MHz}$ & $10 \mathrm{Mbps}$ & PSM & \multirow{3}{*}{ High } \\
\hline & & $4 \mathrm{G}$ & $<100 \mathrm{~km}$ & LTE Band & $5-20 \mathrm{MHz}$ & $100 \mathrm{Mbps}$ & High & \\
\hline & & $5 \mathrm{G}$ & $<100 \mathrm{~km}$ & Sub $6 \mathrm{GHz}$, mmWave & $100-200 \mathrm{MHz}$ & $10 \mathrm{Gbps}$ & High & \\
\hline
\end{tabular}

require factory automation and monitoring of missioncritical equipment.

3) Wireless local area network (WLAN): WLAN has a coverage area of between 100 and 1000 meters, which typically extends from a floor to a building. WLAN transmits data via radio waves; however, cables are now used to connect the network's backbone. IEEE $802.11 \mathrm{a} / \mathrm{b} / \mathrm{n} / \mathrm{ac}, 802.11 \mathrm{af}, 802.11$ ah, and $802.11 \mathrm{p}$ are among the technologies supported by WLAN.

- IEEE $802.11 \mathrm{a} / \mathbf{b} / \mathbf{n} / \mathbf{a c}$ standards supported by the routers are ideal for connecting multiple devices on-site while maintaining network security in an IIoT network. These technologies enable both indoor and outdoor dual band radio access points (APs) that are capable of receiving and transmitting in both bands. Moreover, because these technologies operate at low frequencies, they can easily obstruct concrete structures, such as walls and buildings.

- IEEE 802.11 af commonly known as White-Fi, is classified as WLANs. It is applicable throughout a broad spectrum, depending on the application. White-Fi consumes less power and is used to connect devices across a large geographical region where low-speed connections are tolerable.

- IEEE 802.11ah is another WLANs technology, and it is referred to as WiFis Halow or a low-power WiFis. It is employed in a wide range of IIoT applications, including gadgets, appliances, smart sensors, and actuators, both indoors and outdoors. Additionally, it is used in both urban and rural settings. Its popularity is increased by the backhaul aggregation and hotspot. IEEE 802.11p is a member of the WLANs family of standards, which enables increased data rates/bandwidth with reduced latency/delay. It is optimal for IIoT applications such as smart cities and networking.

4) Wireless neighbourhood area network (WNAN): WNAN is a self-organizing and self-healing mobile ad-hoc network. The low cost, low-power RF circuits with multi-channel transmitter and receiver are the key attributes of WLAN, making it ideal for long-range IIoT applications.

- Wireless smart ubiquitous network (Wi-SUN) is an exciting technology for interconnecting sensors, data centers, and bidirectional control systems in a IIoT network. Additionally, it is a self-organizing, self-healing, and flexible technology that utilizes mesh networks to construct dense networks capable of supporting many connections. Additionally, it consumes less energy and offers enhanced security.

- ZigBEE neighbourhood area network (NAN) enables low-power wireless networks to achieve a high level of network functionality and self-healing capability. Additionally, it is extremely reliable, robust, and scalable. The technology is ideal for smart energy, health care, and 


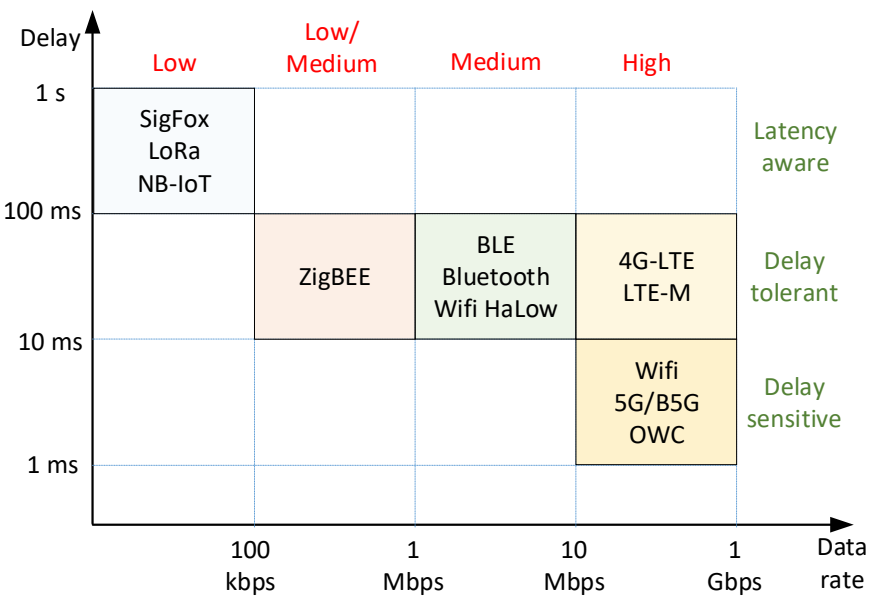

Fig. 6: Data rate vs. latency for various 5G-IIoT communication technologies.

factory/home automation applications.

5) Wireless wide area network (WWAN): WWAN covers an area of up to 100 kilometers. In a WWAN, short packets are transmitted between sensors. It encompasses cellular network technologies such as LTE-MTC, second generation $(2 \mathrm{G})$, third generation $(3 \mathrm{G}), 4 \mathrm{G}, 5 \mathrm{G}$, and $\mathrm{B} 5 \mathrm{G}$, as well as LPWAN technologies such as SigFox, LoRa, Telensa, and NB-IoT.

a) Cellular Networks: The IIoT can communicate over long distances by utilizing an already existing cellular network. Among these cellular technologies are global system for mobile communications (GSM), 3G, and 4G. However, because $4 \mathrm{G}$ is capable of transmitting data over a long distance at a high data rate, it consumes more power. It can be implemented using sensor-based low-bandwidth devices, such as a Raspberry-pi or an Arduino, that are capable of transmitting small amounts of data over the Internet [21], [42]. Recent cellular technologies enable the provision of more sophisticated services for achieving IIoT targets. Several critical cellular technologies for IIoT are discussed in this section.

- 4G standard offers support for long range and high data rate communicaton as compared to $\mathrm{WiFi}$, Bluetooth, White-fi, and Halow for IIoT applications. 4G provides better coverage than $3 \mathrm{G}$, while $3 \mathrm{G}$ provides better as compared to $2 \mathrm{G}$. On the other hand, $2 \mathrm{G}$ consumes approximately $50 \%$ less power than $3 \mathrm{G}$, while $3 \mathrm{G}$ consumes approximately $50 \%$ less power than $4 \mathrm{G}$. These cellular standards enable affordable coverage and data rates in a limited number of IIoT applications, such as mines, warehouses, and manufacturing industries.

- LTE-MTC is a low-power wide area network technology developed by 3GPP and classified as WWAN. LTE-MTC provides a higher data rate with increased mobility and battery life at the expense of increased bandwidth. LTEMTC is typically used in IIoT applications that require low power, increased coverage, and minimal complexity. Among these applications are smart grid and future machine to machine (M2M) networks. The technology operates under power saving mode (PSM).
- 5G/6G key objectives are largely consistent with the IIoT requirements and are as follows: 1) URLLC: delivering high reliability and low latency to time-critical, real-time mission-critical applications such as disaster management. 2) eMBB: significantly increase the bandwidth and speed of smart city applications. 3) glsmmtc: connects millions of devices over long distances [61], [68]-[71] Thus, the key objectives of B5G-IIoT are summarized as follows:

- URLLC, for real-time communication.

- eMBB and mMTC are used to increase the bandwidth and connectivity of a large number of devices.

- Security through network slicing.

- Edge computing is used to ensure proper edge functionality and to share local computations with edge devices.

b) LPWAN: The prime objective of 5G/B5G-IIoT is to provide a long-range, cost-effective communication infrastructure with minimal power consumption. LPWAN is an intelligent network option for connecting multiple IIoT applications. The advantageous features of LPWAN, such as long range communication, increased coverage, low power consumption, and increased throughput/data rate, make it an acceptable solution for IIoT. It is a critical technology for the implementation of B5G-IIoT [26], [53], [72]-[75].

- SigFox widely considered to be the leading international IIoT technology because it enables massive connections to millions of devices and data transfer without the need for a predefined network [49], [76], [77]. It is a widely used wireless technology that bridges the gap between WiFi and cellular networks. Additionally, it significantly improves EE via software-assisted communication. As a ultra narrow band (UNB) technology, it supports low data rates. As a result, it provides a reliable, energyefficient network solution for connecting millions of devices spread across a large geographical area. Typically, this area spans several kilometers, making SigFox ideal for IIoT applications such as smart meters, environmental sensors, and security devices.

- LoRa is another well-known B5G-IIoT technology that is listed under LPWAN. It is used to transmit secure data over long distances of up to several kilometers while using little power. Numerous IIoT networks utilize LoRa to provide services over a larger area than cellular networks. It operates at a wide range of frequencies between $868 \mathrm{MHz}$ and $915 \mathrm{MHz}$. LoRa integrates easily with existing network infrastructure that requires lowpower IIoT applications. It is well suited for IIoT applications such as smart cities that require broad coverage with low power consumption, low cost, and bidirectional communication [41], [58].

- Telensa is an LPWAN technology that enables the deployment of sensors and actuators as a hardware platform for software-defined services. It is suitable for IIoT applications such as smart cities, where smart street lights are regarded as low-cost and low-power sensors on a global scale. 
- NB-IoT is an LPWAN subtype of WWAN that was introduced with the $3 \mathrm{GPP}$ release 13 standardization. NBIoT develops NR technology for IIoT applications such as smart home, smart city, and security systems. Additionally, due to its low power consumption characteristics, it offers promising solutions for future generation wireless technologies. It is a complementary solution to the B5G, URLLC, and mMTC requirements, which are required for the IIoT attributes.

\section{STANDARDiZATION OF URLLC AND EMBB IN $5 \mathrm{G}-\mathrm{IIOT}$}

IIoT requires stringent QoS requirements in both wired and wireless environments, including throughput, latency, reliability, coverage, power efficiency, and mobility [140], [141]. The enabling features and services provided by $5 \mathrm{G} / \mathrm{B} 5 \mathrm{G}$, such as URLLC, eMBB, and mMTC, can assist IIoT in achieving the aforementioned QoS requirements. As a result, B5G-IIoT is regarded as an optimistic solution. As a result, we classify technologies according to the QoS requirements of IIoT in URLLC and eMBB. This section discusses technologies in terms of standardization.

Table III summarizes the key enabling communication technologies. The key enabling technologies in B5G-IIoT which have been aided by URLLC are multi-RAT, 4G, LTE, 5G/B5G, and V2X. Multi-RAT and CoMP, 4G, and LTE are all included in the B5G standard as latency reduction techniques for URLLC. These techniques are based primarily on $\mathrm{mm}$ Wave communication, ultra dense small cell network (UDN), and 3GPP Release 16 in device to device (D2D). Similarly, $\mathrm{V} 2 \mathrm{X}$ is an application-oriented communication technique that includes vehicle-to-vehicle (V2V), vehicle-to-network (V2N), and vehicle-to-infrastructure (V2I) communications. V2X adheres to multiple protocols to reduce latency and increase reliability, as well as to ensure compatibility with missioncritical IIoT applications. Similarly, 3GPP Release 15 and 16 employ a range of licensed and unlicensed spectrum techniques, such as LPWAN and WWAN, to enable the use of B5G-NR, UDN, 4G-LTE, and 5G new waveforms to deliver attractive multi-Gigabit data rates and multi-point connectivity to industry end consumers.

B5G-NR specifies three primary objectives, namely increased network density, increased SE, and access to high spectrum bands. Nonetheless, eMBB integrates multiple techniques, such as B5G-NR communication with UDN, mmWave, and FD communication. mm-Wave communication supports a wide variety of IIoT applications, including timesensitive ones, with a data rate of up to Gbps. In Fig. 6, we compare various B5G-IIoT communication technologies in terms of their data rate and latency requirements. 5G/B5GIIoT aims to provide industrial infrastructure with flexibility and scalability. As a result of the extremely low latency, high reliability, and high throughput of IIoT, it is more effective in automation control and monitoring systems. Following that, this section discusses technologies and their standardization. The key technologies in 5G standardization that can assist in 5G-IIoT compatibility are illustrated in Fig. 7.

\section{A. 3GPP Release 15}

In Release 15, the first phase of the $5 \mathrm{G}$ system is referred to as New Radio, or NR. With this 5G-NR standard, eMBB and eMBB can operate at frequencies up to $52.6 \mathrm{GHz}$. Release 15 of RAT release will include support for both non-standalone (NSA) and standalone (SA) connectivity. The Release 15 system will include critical features such as network slicing, access and mobility management, a QoS framework, a policy framework, and network sharing, as well as the interoperability of unauthorized 3GPP networks, i.e. WiMAX, WiFi, and integration with evolved packet system (EPS). The 5G system architecture and procedures will be described in detail in Release 15 specifications.

There is discussion of 3GPP Release 15, which established the basic framework for URLLC in NR, primarily in support of IIoT. Powered by B5G networks, the IIoT enables new value creation and significantly increases revenue opportunities for original equipment manufacturers (OEMs). IIoT will improve efficiency through industrial automation, while also utilizing a highly flexible, scalable, and virtualized infrastructure. URLLC is based on Release 15 of the 3GPP specification. Two critical requirements for URLLC have been specified in 3GPP:

- Target user plane latency of $0.5 \mathrm{~ms}$ for the downlink (DL) and uplink (UL).

- Packet accuracy of $99.999 \%$ for a 32 -byte packet with a 1 ms user plane latency.

Satisfying URLLC specifications is extremely difficult due to the coupling of high accuracy and low latency. To accomplish this, 3GPP Release 15 defines two distinct physical layer (PHY) feature groups for URLLC support.

The first group contains PHY features that are used to reduce latency. Additionally, features such as flexible slot structure, flexible scheduling, and fast are included. In the second group, PHY features are used to increase reliability. The following features pertain to data enhancements:

- To improve reliability, identical data packets are transmitted from multiple transmission points. This is accomplished through the use of the packet data convergence protocol layer, which duplicates the data packet. It can be used to send the same packets to the user equipment (UE) from two different next generation nodeBs (gNBs) to improve reliability. Repetition with multiple data channel slots - Two, four, or eight repetitions can be used to increase reliability.

- The URLLC enables extremely high reliability through the use of special modulation and coding values.

The major characteristics of NR Release 15, which enables eMBB (10 Gbps - 20 Gbps), are as follows: Ultra-wide bandwidth (up to $100 \mathrm{MHz}$ in $<6 \mathrm{GHz}$, up to $400 \mathrm{MHz}$ in $>6 \mathrm{GHz}$ ), multiple numerologies for optimal operation at various frequencies, native forward compatibility mechanisms, new channel coding, native support for low latency and ultra reliability, flexible and modular RAN architecture that supports split fronthaul, split control- and user-plane, and native endto-end network slicing support. 
TABLE III: Enabling technologies for URLLC and eMBB in 5G-IIoT.

\begin{tabular}{|c|c|c|c|}
\hline $\begin{array}{l}\text { Services for } \\
\text { 5G/B5G- } \\
\text { IIoT }\end{array}$ & Enabling Technologies & 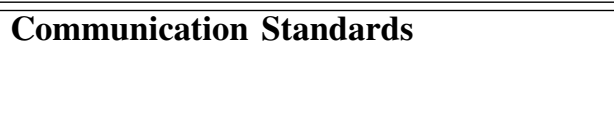 & References \\
\hline \multirow{5}{*}{ URLLC } & $\begin{array}{l}\text { vehicle-to-everything (V2X) communica- } \\
\text { tion }\end{array}$ & 5G/B5G, NB-IoT & {$[78]-[90]$} \\
\hline & $\begin{array}{l}\text { Multi-radio access technology (RAT): co- } \\
\text { ordinated multi-point transmission and re- } \\
\text { ception (CoMP), 4G-LTE,WiFi, 5G NR }\end{array}$ & LTE-M, WiFi/WiFi Halow, NB-IoT & [37], [51], [91]-[97] \\
\hline & Instant and reserved scheduling & LoRa, NB-IoT, cellular & [34], [98] \\
\hline & 5G/B5G, 5G/B5G-NR & Cellular, LPWAN & [99]-[103] \\
\hline & Packet and frame structure & LoRa, B5G & $\begin{array}{l}{[51],[51], \quad[104]-} \\
{[110]}\end{array}$ \\
\hline \multirow{6}{*}{ eMBB } & B5G, B5G-NR & NB-IoT, LoRa & {$[111]-[117]$} \\
\hline & Flexible data-rate (FD) communications & $\begin{array}{l}\text { WWAN, WiFi/WiFi Halow, Bluetooth, } \\
\text { ZigBee }\end{array}$ & {$[112],[118]-[122]$} \\
\hline & NW techniques & B5G & $\begin{array}{l}{[25],[51], \quad[106],} \\
{[123]-[129]}\end{array}$ \\
\hline & Unlicensed spectrum based techniques & LTE-M, NB-IoT, LoRa, Talensa, Sigfox & {$[28],[29]$} \\
\hline & Dense small cell network & $\begin{array}{l}\text { WWAN, WiFi/ WiFi Halow, Bluetooth, } \\
\text { ZigBee }\end{array}$ & {$[130]-[134]$} \\
\hline & millimeter wave (mm-Wave) & B5G & $\begin{array}{l}\text { [51], [111], [135]- } \\
{[139]}\end{array}$ \\
\hline
\end{tabular}

\section{B. 5G Evolution: 3GPP Release 16}

The enhancements to 5G-NR in $3 \mathrm{GPP}$ release 16 include both extensions to existing features and new verticals and deployment scenarios. Unlicensed spectrum operation, intelligent transportation systems, IIoT, and extraterrestrial networks are a few of the highlights [142], [143].

Among them is the capabilities of $5 \mathrm{G}$ to support a large number of mobile and fixed IIoT devices with varying speeds, bandwidths, and QoS requirements. With the expansion of IIoT, the flexibility of $5 \mathrm{G}$ will become even more critical for enterprises that require support for the stringent requirements of critical communications. Due to the ultra-reliability and low latency of B5G, self-driving cars, smart energy grids, enhanced factory automation, and other demanding applications will be possible. As 5G improves network performance, cloud computing, artificial intelligence (AI), and edge computing all contribute to the handling of the data volumes generated by the IIoT. Additional 5G enhancements, such as network slicing, private networks, and $5 \mathrm{G}$ core, will ultimately contribute to the realization of the vision of a IIoT network capable of supporting a massive number of connected devices. The most significant enhancements in release 16 are in the areas of multiple-input multiple-output (MIMO) [144], [145] and beamforming enhancements, dynamic spectrum sharing (DSS) [146], dual connectivity (DC) and carrier aggregation (CA) [147], and power savings in UE [148], [149].

This release is targeted at industrial automation use cases. The 5G-URLLC foundation has been enhanced in Release 16 to ensure more reliable communication of up to $99.99 \%$. Increased retransmissions are insufficient for these use cases, as there is a strict latency constraint as well. CoMP can be used in conjunction with other technologies to overcome this constraint. Multiple transmission and reception architecture (Multi-TRP) establishes redundant communication paths with spatial diversity, ensuring that communication is maintained even if one of the paths is temporarily blocked by using the remaining paths [32], [33]. Moreover, this release addresses new vertical and deployment scenarios, such as integrated access and backhaul (IAB) NR in unlicensed spectrum features related to IIoT and URLLC.

The IIoT is a significant vertical focus of NR Release 16. It includes latency and reliability enhancements on the already existent Release 15's work on high reliability and low air-interface latency. This release expanded IIoT use cases such as factory automation and electrical power distribution. Additionally, there is support for time-sensitive networking (TSN), which is critical for time synchronization. When the URLLC-related improvements are added together, they have a significant effect on NR. Release 16 extends the inter-UE DL preemption support introduced in Release 15 to the UL, allowing a UE to cancel a lower-priority UL transmission in favor of a higher-priority UL transmission from another UE. Additionally, Release 16 supports standardizing the resolution of intra-UE UL resource conflicts. Control-channel monitoring is more frequent in this release to reduce latency. Moreover, multiple configurations for both UL configured grants and DL semi-persistent scheduling can be active concurrently. The enhancements are especially beneficial for TSN traffic, where the base station (BS) is aware of the traffic pattern [150].

\section{5G Evolution: 3GPP Release 17}

The 3GPP release 17 includes support for new use cases, such as public safety, non-terrestrial networks, and private networks, as well as enhancements to existing ones, such 


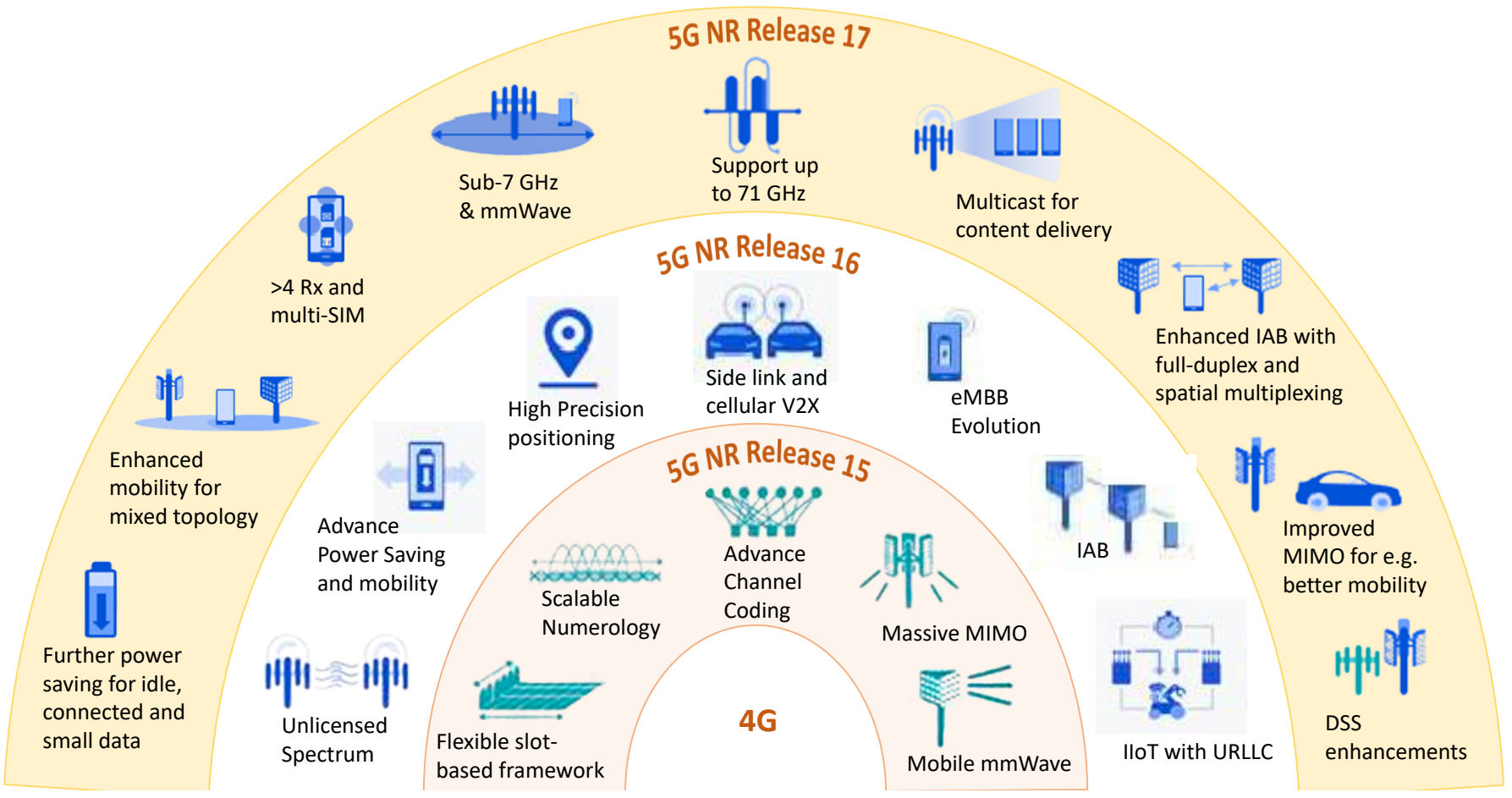

Fig. 7: Techniques in 5G standardization that can assist 5G-IIoT.

as mobile broadband, industrial automation, and vehicle-toeverything. Moreover, this release includes improvements to existing services and use cases, as well as new deployment alternatives and use cases.

Together with previously established use cases and deployment options, 5G will incorporate intelligent network technologies and support a large number of new use cases. One of the important components of $5 \mathrm{G}$ is the application of AI based on machine learning (ML) techniques. The implementation of $\mathrm{AI} / \mathrm{ML}$ in future wireless networks will trigger a paradigm shift. AI and ML will be used to solve multidimensional network optimization problems in an online or offline manner, enabling the introduction of intelligent network management. By optimizing the performance of multi-antenna systems with AI/ML, for example, the radio interface can be enhanced as well. Wireless networks will enable immersive experiences in virtual and physical environments, as well as human-machine interaction with devices and wearables in IIoT, through extended reality communication.

A significant portion of the functionality already implemented in live NR networks will be enhanced in Release 17, which mark the beginning of the transition to $5 \mathrm{G}$ Advanced or B5G.

1) Beamforming and MIMO: Enhancements in Release 17 MIMO focus on four areas: beam management; Multi-TRPs to enable URLLC; Multi-TRPs to enable eMBB; and reciprocity in time division duplex (TDD) and frequency division duplex (FDD).

The goal of streamlining signaling and optimizing operator equipment (UE) with multiple antenna panels is to increase performance while maintaining high mobility. Multi-TRP has been added to the physical downlink control channel (PD-
$\mathrm{CCH}$ ), physical uplink shared channel (PUSCH), and physical uplink control channel (PUCCH). Along with providing more detailed channel state information (CSI) feedback for noncoherent joint transmission, the new algorithms improve performance for high-speed train communications. Enhancements to reciprocity-based operation include the creation of new codebooks with reduced feedback overhead, the availability of channel information at $\mathrm{gNB}$, and enhancements to the sounding reference signals.

2) Dynamic Spectrum Sharing: Using the DSS included in Release 15, it is now possible to deploy both a LTE and a NR cell from the same BS. Release 16 primarily increased the capacity of the physical downlink shared channel (PDSCH). Moreover, the release enables operators to better manage PDCCH resource shortages in the NR cell that occur as the number of NR UEs increases. Cross-carrier scheduling, introduced in Release 17, schedules data channels on the shared primary cell via the PDCCH of a DL secondary cell.

3) User Equipment Power Savings: Release 17 includes power-saving enhancements for UEs operating in connected, idle, or inactive radio resource control (RRC) modes. Both eMBB UEs and RedCap devices are specified with powerefficiency enhancements. Several power-saving enhancements are included in this release's features, including relaxed radio resource monitoring for devices operating at low mobility or under ideal radio conditions, extended discontinuous reception for devices with a low latency tolerance, reduced PDCCH monitoring during active time, and power-efficient paging.

4) Positioning: NR added support for positioning via LTE (for non-standalone deployments) and RAT-independent positioning (Bluetooth, wireless LAN, pressure sensors, etc) in Release 15. A new version of Release 16 introduced time- 


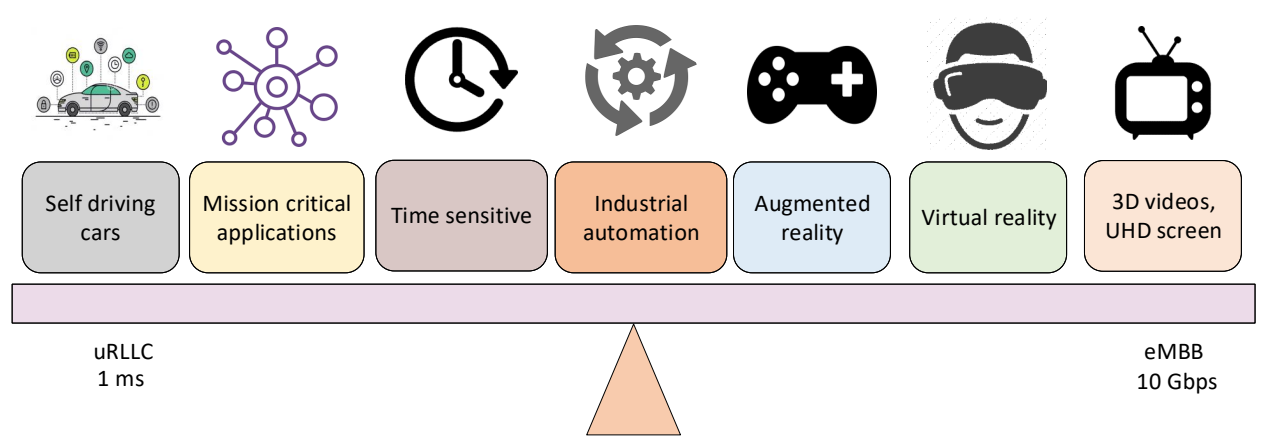

Fig. 8: Trade-off between URLLC and eMBB.

based positioning methods for NR standalone deployments (multi round trip time (RTT), the DL time difference of arrival, and the UL angle of arrival), which can be used in conjunction with timing-based solutions to improve accuracy.

Release 17 further improves NR positioning for specific use cases such as factory automation by aiming for 20-30 $\mathrm{cm}$ accuracy in certain deployments. Additionally, Release 17 improves latency reduction, enabling positioning to be accomplished in time-critical applications such as remote control. Along with high-accuracy positioning, the integrity of location information is critical in IIoT and automotive use cases. The purpose of this release is to introduce key performance indicators to demonstrate the reliability/integrity of a measurement report that addresses the global navigation satellite system (GNSS) positioning procedure.

5) Ultra Reliable Low-Latency Communication: Using URLLC, 5G has penetrated into multiple vertical markets. Release 15 established a strong foundation, and Release 16 added enhancements by the 3GPP's system architecture and RAN groups to improve support for vertical industries such as factory automation, shipping and transportation, and electrical power distribution. Several redundancy schemes were added to the user plane as part of these enhancements, as well as improvements to reliability, latency reduction, and support for time-sensitive communications (TSC). Enhancements in Release 17 are intended to increase SE and system capacity, while also supporting URLLC in unlicensed spectrum environments and strengthening the framework for supporting TSC. Additionally, enhancements to hybrid automatic repeat request (HARQ), CSI, intra-UE multiplexing, and service survival time will be included in the assistance information provided by the TSC.

6) NR coverage: The coverage of a company or organization has a direct impact on service quality, operating expenses, and capital expenditures, making it a critical factor in commercialization and competition. The PUSCH is identified as a potential coverage bottleneck in Release 17. As part of the 3GPP initiative to expand PUSCH coverage, mechanisms for transport block processing repetition and support for multiple slots are being investigated. Additionally, Release 17 specifies mechanisms for grouping demodulation reference signals (DMRS) and indicating dynamic PUCCH repetition factors.

7) Small Data Transmission: The existing NR RRC inactive mode promotes power-efficient connection establishment by allowing UEs to resume a previously established RRC connection. According to Release 17, data transmission in RRC inactive mode further reduces the power consumption of UEs during system access. This is especially important for low-power devices that transmit data infrequently and in small amounts in order to minimize control plane signaling overhead by avoiding the need to resume an RRC connection.

8) Non-Public Networks: 3GPP Release 16 defined nonpublic networks (NPN) as those that provide access to a subset of users, such as devices belonging to a particular factory. The 3GPP specified two options for NPN deployment as part of its support for industrial verticals. An NPN that is integrated into the public network enables operators to support NPNs by directly integrating them into their networks. A standalone NPN is another option for deployment. As a general rule, a standalone NPN is functionally and characteristically comparable to a standard public network. Standalone NPN receive additional enhancements in 3GPP Release 17. The enhancements include the ability for a UE to attempt access to a standalone NPN using external credentials (such as those from a public network or another standalone NPN), UE onboarding to the standalone NPN (for example, by providing it with NPN credentials and/or subscription parameters), and support for emergency services.

9) Edge Computing: Release 15 of $3 \mathrm{GPP}$ enables operators and third parties to host services close to the UE's access point of attachment. The baseline architecture reduces endto-end latency and the load on the transport network. In Release 17 , mechanisms for discovering edge application servers are introduced. For instance, it defines an edge application server discovery function (EASDF) whose primary purpose is to facilitate the session breakout connectivity method. In addition to acting as a domain name system (DNS) resolver, the EASDF can respond to DNS queries with UE location information. The UE locations can then be resolved to nearby application servers via the DNS.

Release 17 clarifies and enhances the use of user-equipment route-selection policy (URSP) rules in the distributed anchors and multiple protocol data unit (PDU) session connectivity models. When configuring URSP rules in the UE, application server information can be taken into account. As a result, the UE can establish PDU sessions dynamically for specific application servers, obviating the need for complex session breakout solutions. Additionally, Release 17 introduces new 
mechanisms for exposing QoS monitoring results and enhances support for application server relocation in the event of a UE's mobility.

\section{TRADE-OFF BETWEEN URLLC AND EMBB FOR 5Gs/B5G-IIOT}

B5G-IIoT features various use cases related to high definition (HD)-video, virtual reality (VR), augmented reality (AR), smart cities, smart cars, smart factories, smart grid, remote control and autonomous robots and AI networks. These use cases possess sensors, actuators and processors along with massive IIoT applications. However, EE, SE, latency, reliability and throughput requirements vary for different IIoT applications. ITU has defined the QoS requirements for URLLC, mMTC, and eMBB in B5G standard. For URLLC, a reliability of $99.99 \%$ and less than $1 \mathrm{~ms}$ latency is required. $\mathrm{EE}$ and connection of massive IIoT devices is a parameter for mMTC, and throughput of up to Gbps is desired for applications categorized under eMBB. Therefore, B5G provides effective services to the IIoT applications and their corresponding use cases under varying QoS requirement. However, use cases which achieve URLLC suffers with a degradation of eMBB. On the contrary, if eMBB is achieved URLLC would be degraded. Therefore, a trade-off between the two is of particular emphasis, i.e., to achieve URLLC and eMBB simultaneously with same resource efficient method to satisfy the QoS requirement defined by ITU. Thereby, the coexistence of URLLC and eMBB open the doors to new technologies and research directions [31], [36], [174]-[178].

\section{A. Performance Trade-off between URLLC and eMBB}

Design trade-off between URLLC and eMBB is widely investigated by the research community. Some key trade-offs determined by the researchers are discussed herein.

1) Finite- vs. large-block length: In the recent past, the study of the effective capacity of fading channels has caught the attention of many researchers in order to understand the performance of wireless systems under the influence of statistical queuing. Although channel coding is performed using a finite block of symbols, in almost all prior studies the service rates of the queuing model were assumed to be equal to the instantaneous capacity values. In addition, transmissions are assumed to be error-free with no decoding errors. It is important to note, however, that error-free communication at the rate of channel capacity can be achieved as codeword length increases without limit. In order to have high reliability or lower error probability, finite block length codes will necessarily use coding errors and will transmit at a lower rate than the channel capacity. However, due to noise, deep fading, collisions, and interference, low-latency applications with small block lengths are prone to failure. If $k$ information bits are transmitted using coded packets over $n$ channel uses, the maximum coding rate $R=\frac{k}{n}$ is lower than the Shannon rate. Additionally, in order to ensure high reliability, encoded data must be transmitted at a lower rate than Shannon's capacity. With a large blocklength, no errors occur and the additive white gaussian noise (AWGN) channel's achievable rate is equal to Shannon's known capacity $R=\log _{2}(1+\gamma)$, where $\gamma$ is the achieved signal to noise ratio (SNR). Shannon's capacity model has been shown to significantly overestimate delay performance for these applications, resulting in an insufficient allocation of resources. A theoretical framework is needed to model the performance of such systems due to short time spans, finite block lengths, and interference despite the huge interest in the field [179], [180].

2) Spectral efficiency vs. latency: Additionally, transmission schemes must be capable of supporting a significant number of devices within a given bandwidth while maximizing battery life. With this approach, effective bandwidth has the potential to provide a good balance between SE and transmission time for a given coverage. To avoid excessive transmission time, the allocated bandwidth for a device should not be much less than the effective bandwidth. To be able to accommodate more B5G-IIoT devices while utilizing the available bandwidth without significantly increasing transmission time, it is preferred to allocate bandwidth that is not greater than the device's effective bandwidth for a given sensitivity level. Due to the short transmission time interval (TTI), low latency incurs a SE penalty. SE and latency are not characterized in a multiple access and broadcast system that takes into account: a) bursty packet arrivals; b) traffic that is low latency and delay tolerant; and c) channel fading and multipath. To effectively support the huge number of B5G-IIoT devices in 5G and beyond cellular networks, advanced transmission scheduling techniques and low signaling overhead medium access control (MAC) protocols need to be investigated [181].

3) Energy efficiency vs. latency: An important trade-off that needs to be identified is energy consumption versus latency. The battery life of B5G-IIoT devices is extended by placing them in sleep mode or deep sleep mode while they are not in use. The device needs to check periodically for packets sent by applications on the network, since applications sending packets to the device may wake it up. In addition to the latency of the packets, device check frequency, determining how much energy is consumed. As the frequency increases, the latency will decrease, but energy consumption will rise. When reliability deteriorates, re-transmissions are necessary, which means more energy is consumed to provide a target level of reliability. Energy is measured generally using $\sqrt{d^{-1}}$ or $\sqrt{d^{-1} \log d}$, where $\mathrm{d}$ is the delay. This means that the energy decreases quite slowly with increasing delay [182].

4) Reliability vs. latency vs. data rate: IIoT and nextgeneration wireless communication standards, including $5 \mathrm{G}$ and $\mathrm{B} 5 \mathrm{G}$, provide applications which requires rethinking the communication stack, practical codes, networking architecture, and control design to provide ultra-low latency of $1 \mathrm{~ms}$, extremely high reliability of more than $99.99 \%$, and $20 \mathrm{Gbps}$ peak data rates. However, higher reliability comes at a cost of higher latency. Typically, the reliability is ensured with retransmissions which induce additional delays. However, higher data rates is achieved at the expense of lower reliability, and vice versa.

5) SNR vs. diversity: Diversity is typically defined when plotted against the SNR on a logarithmic scale as the slope of probability of error. The key question is: how does the SNR 
TABLE IV: URLLC and eMBB based B5G-IIoT applications; Lat.: Latency, Rel.: Reliability, Tp.: Throughput, Cell dens.: Cell density.

\begin{tabular}{|c|c|c|c|c|c|c|}
\hline $\begin{array}{l}\text { Smart ap- } \\
\text { plications }\end{array}$ & Use cases & Lat. (ms) & Rel. & $\begin{array}{c}\text { Tp. } \\
\text { (Mbps) }\end{array}$ & $\begin{array}{l}\text { Cell dens. } \\
\left(\mathrm{Gbps} / \mathrm{km}^{2}\right)\end{array}$ & Ref. \\
\hline \multirow[t]{6}{*}{ Factories } & Printing \& packaging machines & $2 \& 1$ & $10^{-9}$ & $1,<100$ & 10 & [19], [151]-[163] \\
\hline & $\begin{array}{l}\text { Machine tools \& manufactur- } \\
\text { ing }\end{array}$ & $0.5 \& 5$ & $10^{-9}$ & $<100$ & $<10$ & [151], [156], [163] \\
\hline & $\begin{array}{l}\text { Monitoring automation \& re- } \\
\text { mote control }\end{array}$ & 50 & $10^{-3}-10^{-5}$ & $1,<100$ & $<10$ & $\begin{array}{c}{[19],[156]-[162],[162],} \\
{[163]}\end{array}$ \\
\hline & $\begin{array}{l}\text { Remotely operated video \& } \\
\text { motion control }\end{array}$ & $\begin{array}{c}10-100 \& \\
1\end{array}$ & $10^{-9}$ & $<100$ & 14 & {$[152]$} \\
\hline & Industrial robots & $4-8$ & $10^{-9}$ & $<100$ & 10 & {$[164]-[167]$} \\
\hline & Milling machines \& cranes & $4-8 \& 12$ & $10^{-9}$ & $1,<100$ & 10 & {$[164]-[167]$} \\
\hline \multirow{3}{*}{$\begin{array}{l}\text { Autonomous } \\
\text { vehicles }\end{array}$} & Accidental warnings & 10 & $10^{-3}-10^{-5}$ & $0.1-29$ & $\mathrm{~N} / \mathrm{S}$ & [79], [162], [168], [169] \\
\hline & High speed train & 10 & $\mathrm{~N} / \mathrm{S}$ & $25-50$ & $12.5-25$ & [19], [154], [155] \\
\hline & Automated driving & 5 & $10^{-5}$ & $0.1-29$ & $\mathrm{~N} / \mathrm{S}$ & [19], [154], [155] \\
\hline \multirow[t]{2}{*}{$\begin{array}{c}\text { Grid } \\
\text { Stations }\end{array}$} & Medium electricity distribution & 25 & $10^{-3}$ & 10 & 10 & [19], [154], [155] \\
\hline & High electricity distribution & 5 & $10^{-6}$ & 10 & 100 & [19], [154], [155] \\
\hline \multirow[t]{5}{*}{ Cities } & Outdoor industrial events & $1-2$ & $10^{-2}$ & 30 & 900 & [155], [162], [168]-[173] \\
\hline & Public gatherings & $10-100$ & $10^{-2}$ & $0.3-20$ & $\begin{array}{c}100- \\
10000\end{array}$ & [155], [162], [168]-[173] \\
\hline & Shopping centers & $10-100$ & $10^{-2}$ & $60-300$ & 60 & {$[155],[162],[168]-[173]$} \\
\hline & Mass communication & $200-5000$ & $10^{-2}$ & 15 & 60 & {$[155],[162],[168]-[173]$} \\
\hline & Urban area & $1-2$ & $10^{-2}$ & $60-300$ & 700 & {$[155],[162],[168]-[173]$} \\
\hline \multirow{5}{*}{$\begin{array}{c}\text { Traffic } \\
\text { manage- } \\
\text { ment }\end{array}$} & Traffic congestion & 8 & $10^{-3}$ & $20-100$ & 480 & {$[154],[155]$} \\
\hline & Traffic management efficiency & $<100$ & $10^{-3}$ & 10 & 10 & [19], [153]-[155] \\
\hline & Urban crossings & $<100$ & $10^{-5}$ & 10 & 10 & $\begin{array}{c}\text { [19], [153]-[155], } \\
{[168]-[173]}\end{array}$ \\
\hline & Safety measures for highway & $10-100$ & $10^{-3}-10^{-5}$ & 10 & 10 & {$[19],[153]-[155]$} \\
\hline & safety measures for urban area & $10-100$ & $10^{-3}-10^{-5}$ & 10 & 10 & [19], [153]-[155] \\
\hline
\end{tabular}

requirement decrease with increased diversity and nodes in the network? How does the use of higher frequency bands affect diversity? It's important to know how much SNR is needed to balance time-varying channels, and bad fading events. SNR significantly effects the diversity but compensates the time varying channels.

6) Open vs. closed loop: In closed loop system low data rates are obtained due to the utilization of limited resources. In an open loop, the cooperative devices, like the relay nodes, broadcast the packets for transmission which results in a reduced delay. Moreover, the diversity puts a positive impact on the reliability. Multiplexing techniques, such as open-loop MIMO, maximize multiplexing gains. Despite this method's ability to transmit multiple data streams using multiple transmit antennas, it requires a complicated detection algorithm in the receiver. Unlike open-loop MIMO, closedloop MIMO techniques build capacity or improve SNR based on channel knowledge. Since closed-loop MIMO in highly mobile environments experiences a delay between transmitting and receiving channel information, designers should be careful when using it. In addition, MIMO with closed-loop feedback and limited uplink sounding performs poorly due to incomplete channel knowledge.

\section{B. Enabling Technologies and Standards for Trade-off be- tween URLLC and $e M B B$}

Many existing enabling technologies cope with the trade-off between URLLC and eMBB. However, this is still an open research question to obtain an optimal trade-off point.

1) TTI: The TTI in $5 \mathrm{G} \mathrm{NR}$ and LTE is reduced to $1 \mathrm{~ms}$ and $0.125 \mathrm{~ms}$, respectively, by introducing wider spacing between sub-carriers. Further improvement is realized by reducing the RTT during HARQ retransmissions, eventually leads to a significant reduction in latency. To satisfy the QoS constraint of reliability, minimum time should be consumed during HARQ retransmissions. Having said that, the reduction in symbol duration and growing spaces between the sub-carriers also lessen the achieved rate and available resources for the transmission. Therefore, a trade-off is imperative between the latency and sub-carrier resources.

2) Multiplexed $e M B B$ and URLLC: For an efficient and optimized system, the resource partitioning is static or semistatic between eMBB and URLLC. Typically, high frequency is required to achieve high degree of reliability besides low latency. However, intelligent and optimized scheduling schemes are used to transmit low latency packet, while relaxing the eMBB. 
3) Network slicing and edge caching: Edge caching and cloud computing have shifted and shared the network load to the edges, thereby, reduced the latency of URLLC. In parallel, additional bandwidth and caching resources to resource are gained through network slicing. These techniques have provided a boost to the sensitive applications like AR and VR.

4) $M L \& A I:$ ML has gained an overwhelming attention of the scientific community during the last decade. Typically, the ML executes the global data sets for classification and regression to approach a solution. ML, together with AI, has delivered a notable gain to the QoS requirements of URLLC by solving a problem in a distributed fashion, and also referred to as $\mathrm{AI}$ and ML on the edge and on the device.

5) Grant free access: In persistent scheduling, rapid access to the resources on high priority is mandatory. However, in semi-persistent unused resources must be allocated to the eMBB applications. In grant based access, the BS control the resources. However, in grant free access, the resource assignment procedure is skipped which eventually enhancing the latency of URLLC.

\section{Applications of 5G-IIOT IN-TERms of URLLC to EMBB}

With the emergence of 5G/B5G, several IIoT applications such as smart cities, smart industry to smart grid and intelligent networks gain benefits from its distinguished features, e.g., URLLC and eMBB are two notable services for IIoT applications. eMBB fulfils the bandwidth requirement of data-intensive IIoT applications, e.g., AR, industrial video surveillance and VR. However, applications such as robotics, motion control, autonomous driving controls are time sensitive and mission critical applications and are, therefore, served by URLLC. Moreover, eMBB provides much higher data rate of up to Gbps with better coverage, while URLLC reduces the delay and provides the latency of $1 \mathrm{~ms}$ to the IIoT applications. Table IV provides IIoT applications along with their throughput, latency, and reliability bounds.

Various B5G-IIoT applications are shown in Fig. 8. These applications are self driving car, mission critical application, i.e., remote control robots and machines, time sensitive applications in which latency is a grave concern, industry automation AR, VR and HD-video. These applications are categorized from URLLC to eMBB. Each application has its own QoS requirement which needs to be satisfied via different enabling and communication technologies. These important IIoT applications are discussed herein.

\section{A. Smart Factory}

Smart factory design parameters are mission-critical, with closed-loop control system which requires a latency of less than $1 \mathrm{~ms}$ and $99.99 \%$ service availability. Specifically, industrial and health robots with motion control are categorized as URLLC, as they are delay sensitive and requires high reliability and low latency. However, eMBB based AR and VR need high data rate and throughput. Therefore, in general B5G-IIoT provides communication between inter and intra industrial factories.
Certain use cases for smart factories are discussed in Table IV with corresponding parameters. These use cases include printing and packaging machines, remote control automation and monitoring, industrial robots, video and motion control, milling machines and cranes. Moreover, the latency and throughput requirements of the aforementioned use cases are also listed. URLLC use cases include automation, industrial robots, motion control [151]. These use cases seeks a latency of less than $10 \mathrm{~ms}$ and $99.9999 \%$ resources availability. While eMBB applications include virtual and augmented reality which needs high data rate universal connection [19], [152], [156], [163]-[167].

Smart Factory is an application of B5G-IIoT. To get maximum benefits from it, the key implementation and QoS maintaining issues are discussed. Smart factories have many interconnected networks, industries, and devices including industrial wireless sensor networks (WSNs) which needs connections with minimal delays, must be power efficient for continuous processing, and provide no queuing of data. To jointly exploit all these services and features in a smart factory is a big challenge for the research community. Licensed and unlicensed spectrum is used for communication between devices to gain maximum possible data rates. In B5G, the $\mathrm{mm}$-Wave is considered a suitable choice for high throughput, reliability, and low latency. However, it suffers from various attenuation.

\section{B. Smart Autonomous Vehicles}

Smart vehicles require an efficient real-time exchange of data acquisition and processing. Smart vehicles are designed with many sensitive sensors which can be efficiently controlled and monitored to fulfill the requirements of URLLC and eMBB. The efficient control of sensors ensures the safe exchange of real-time secure transfer of large volume of data at high rate. The latency requirement of less than $10 \mathrm{~ms}$ and a throughput of up to Mbps encourages to partially or fully shift the data processing to the cloud and the edge. To design a smart autonomous vehicle system, data sensing and transmission can be done from vehicle to vehicle and from vehicle to traffic using diverse communication technologies including ZigBee, WiFi, and 5G/B5G.

Several smart autonomous vehicle use cases are discussed in Table IV. These use cases are accidental warnings, highspeed trains, and automated driving. These use cases are timesensitive and need a high-speed data exchange. For example, in an accidental scenario, ultra higher throughput with minimal latency is desired [154], [155], [168], [169].

\section{Smart Grid}

Smart grid is among the most monitored and controlled IIoT applications. Owing to B5G, the smart grid generates, transmits, and distributes electricity with additional reliability, scalability, sustainability and safely. From consumer perspective, the usage of electricity is more adaptive in high speed vehicles, storage units, wireless sensors and IIoT devices. B5G-IIoT drives the smart grid more efficiently due to reduced delays and enhanced reliability. Moreover, B5G-IIoT connects 
low-voltage grid stations to other elements of smart grid, and the backbone networks of B5G to medium and high power voltage grid stations.

Typical use cases for smart grid include medium and high electricity distribution stations, provided in Table IV. These use cases have different latency and throughput requirements according to their targets, i.e., future and sudden disturbance in normal grid station need less than $5 \mathrm{~ms}$ of latency and a data rate of up to $30 \mathrm{Mbps}$ for up-link and $3 \mathrm{Mpbs}$ for down-link receptions. Thus, it is expected that up to 100 customers can be simultaneously supported with less than 1 ms delay and $99.999 \%$ resources availability. Similarly, B5GIIoT uses mm-Wave for smart meters for accurate prediction of the energy usage of the consumers and to effectively transmit the information to the micro-grid [154], [155], [183]. The problems often occurring in the industrial smart grids are required to be timely controlled. Moreover, the seamless power transmissions between city areas need to be real-time, timesensitive, and should be monitored and controlled to avoid disruptions in connectivity.

The Smart Vehicle IIoT applications suffer from wireless connectivity and other QoS related challenges. Some of the challenges like system performance reliability, connectivity, and passenger's internet availability are the main concerns. The process of acquisition and processing of real-time data in a small vehicle, global positioning system (GPS) connectivity, fuel, and hazard monitoring applications need efficient communication, while jitters and latency can cause serious hazardous situations. Thus, real-time data acquisition and timesensitive applications are challenging to deal with. Allocation of resources in a smart vehicle is also challenging because of its mobility and constantly changing geographical locations. Thus, efficient implementation of V2X infrastructure with multi-RAT technology for low latency, high throughput, and high reliability makes it more challenging for smart vehicles.

\section{Smart Cities}

The concept of smart cities is trending from the last decade. Typically in a smart city, the services like, the transportation, health-care and schooling are integrated with the communication infrastructure. Traffic update is another modern time sensitive smart city application and, therefore, can be implemented using URLLC. Thus, IIoT plays a pivotal role in building smart cities by integrating different applications at high data rate, high reliability, and low latency. Communication technologies like LoRa wide area network (LoRaWAN), SigFox, WiFi, 802.11x, Bluetooth and LTE are used data collection and processing of data from smart city sensors'.

Use cases for smart city include managing of larger events like football matches, shopping malls and media. High-quality live coverage is needed for media activation and at ultra higher data rate. Similarly, health and traffic systems seek real-time monitoring and should be communicated with less latency and high reliability to the consumer end. B5G-IIoT supports high reliability, higher data rate with less than $1 \mathrm{~ms}$ latency for smart cities [155], [162], [168]-[173].

Smart Cities has many applications like smart homes, transportation, street lighting. Almost all these applications the QoS requirements of URLLC and eMBB need to be implemented in different ways, using different communication and enabling technologies. Real-Time and time-sensitive monitoring and control applications, including accidental cases, highways, and traffic, are susceptible to low latency and high reliability. However, for large gatherings in shopping malls and stadiums, the real-time coverage with high throughput and low latency is required. Thus, joint optimization of multiple QoS constraints is a challenging task.

\section{E. Smart Healthcare Service}

New opportunities in healthcare improvement are discovered through the IIoT [184], [185]. Owing to ubiquitous identification, sensing, and communication capabilities in IIoT, all objects in the healthcare system can be continuously tracked and monitored [186]. These objects comprise the people, medical equipment, and medicine. Healthcare-related information can be collected, managed, and shared through global connectivity. This information is related to the logistics, diagnosis, therapy, recovery, medication, management, finance, and even the daily activities of the patients. For example, the doctors can periodically monitor the heart rate of a patient under an IIoT aided healthcare facility. The IoT-based healthcare services can be formed as mobile and personalized using personal computing devices, e.g., laptops, mobile phones, tablets, and mobile internet access [187]. The universal availability of mobile internet has accelerated the development of IoT-based at-home healthcare (AAH) services [185]. Security and privacy are the major concerns and challenges in smart healthcare services.

In smart hospitals, B5G-IIoT plays an important role and provides services for living and the health care system. Remote health monitoring of the devices and patients must be quick with minimum latency and high reliability. Similarly, a robot that assists in surgery requires minimum end-to-end latency, high reliability, and throughput which makes it more sensitive and challenging.

\section{F. Smart Homes}

Smart homes are intelligent buildings, equipped with smart devices and sensors that are connected to the internet, are able to make instantaneous and distributed decisions. Besides, the users can control the electrical appliances with smart and intelligent technologies, including bilateral computer interfaces (BCI) and AI. These technologies analyze human behaviors to create a safer, convenient, and easier environment for the end-users. The smart home will become a reality with $5 \mathrm{G}$ and B5G.

\section{G. Smart Robots}

Autonomous robotic systems allow the development of the capabilities of individual robots, their integration into platforms, and enable the development of fleets of heterogeneous robots for collaborative applications. Usually, robotic applications require robust and resilient wireless/cellular communication. The applications use a variety of communication 
channels, including cellular, optical, audio, and video. In communication networks, protocols for many purposes may be used, including 802.15.1, 802.15.4, 802.11, 4G/LTE/5G, and more. Edge-distributed processing, instead of centralized processing, is more suited for mission- and safety-critical robotic applications. These applications include analytic based on AI methods. Edge-distributed processing makes use of multi-access edge and fog computing technologies, as well as wireless and cellular communication, i.e., 4G, 5G and B5G. The use of intelligent networks can facilitate the transfer and processing of information in an energy-efficient manner. The artifical neural networks (ANNs), ML, and other AI techniques further improve the efficiency of decentralized data analytic, automated network management, and context and knowledge sharing for robots and IIoT applications.

\section{H. Smart Industrial Manufacturing}

Industries are always in dire need of smart, efficient, and cost-effective manufacturing solutions to increase their revenues, and simultaneously to fulfill the market's demand and supply needs. Modern technologies including robotics, warehouse automation, and smart factories play a vital role in the industries to accomplish the manufacturing goals. These advancements are further enhanced through B5G wireless networks and IIoT. With B5G, manufacturing industries will be quickly transformed into smart industries by automation, $\mathrm{AI}$, and AR techniques. The B5G revolution in industry digitization is upgrading connectivity, speed, quality, and latency. The B5G can successfully handle the manufacturing challenges, including bandwidth, speed, and latency. Owing to the benefits offered by B5G, factory floor machines and robots will be connected for better predictive maintenance. For factory floor layout changes and adjustments, B5G networks will offer higher flexibility, lower costs, and shorter lead times. With advanced B5G networks and information processing technologies, smart factories can be streamlined, internal and external communications can be improved, and product life cycle management can be unified on a single network.

\section{Smart Forestry}

Digital forestry is turned into smart forestry with characteristics such as perception, materialization, intelligence, and ecology. Research, production, management, and services in forestry are heavily dependent on private wireless communication networks. In the forest environment, wireless connectivity is the main challenge. Typically, distinct wireless communication technologies are used to overcome the challenges in smart forests. These technologies comprise GSM, general packet radio service (GPRS), Zigbee, bluetooth low energy (BLE), IPv6 over low-power wireless personal area networks (6LoWPAN), RFID, LoRa, Sigfox, NB-IoT, LTE, WiFi, NFC, and $\mathrm{Z}$-wave. With the smart forestry, management and service values of forestry will be deeply explored and the forestry economy will become more prosperous.

\section{J. Smart Traffic Control}

Smart traffic management is susceptible to low latency and high reliability for proper management and observation of traffic, between cities and on highways. Data exchange must be with high reliability and low latency to reduce hazards and emergencies. URLLC and eMBB plays an important role in road safety management [19], [153]-[155].

\section{Future RESEARCH DiRECTIONS}

Despite the promises IIoT offers, there are challenges that needs to be addressed to utilize the full benefits. To fulfill the requirements of industry, some of the research gaps are identified and discussed in this section. The major future research topic in 5G/6G-IIoT are presented in [26], [44], [49], [74], [188]. Following are some of the research directions for IIoT that needs attention

\section{A. Dense IIoT Applications}

In B5G-IIoT, small applications are interconnected to make IIoT network. Research studies are required to make a dense IIoT network with feasible network infrastructure and integration of communication technologies in B5G-IIoT. B5G-IIoT refers to a network of connected objects that form a network of interconnected smart factories. Hardware refers to the physical existence of devices, while software refers to the capability of IIoT applications to provide services for everyone at the same time at different locations. It is not common for industrial devices, such as sensors, actuators, and controllers, to run learning algorithms. Nevertheless, smart industrial devices can potentially provide learning platforms by leveraging their computing capabilities. Learners can run learning tasks on those platforms that require fewer resources (e.g., computing, energy, etc.). By enabling the learning on industrial devices, the intelligence and performance of IIoT can be improved. A practical approach is to design a IIoT device in-loop with simulation platform for the internet of everything, which integrates computer simulations with IIoT device test beds. Simulating IIoT hardware testbeds from real-world data is what makes IIoT device in-loop simulation feasible. A further avenue is to develop an integrated simulation framework for IIoT that can capture the interactions and reciprocal effects between cyber and physical systems.

\section{B. Integration of Cloud and Edge Computing}

In B5G-IIoT, computing is a critical factor that affects latency, reliability, and other performance metrics. In order to provide storage, transmission, and analysis of industrial data, IoT requires powerful and efficient computing platforms. In cloud computing, the computing resources are leveraged from the cloud to assist an industrial unit, on the other hand edge computing leverage computing resources from network edge devices. The hybrid cloud and edge computing platform provides IIoT computing infrastructure with the required capability and efficiency. Due to limitations on complexity, storage, and processing power, it may be impossible to carry out training on a device for a sufficiently complex learning problem. By reducing network latency through edge computing, it will be possible to cut the effectiveness of the learning process. Integrating edge computing infrastructures, especially 
in an industrial context, improves resource utilization. Edge computing, single and multiple edge and cloud connectivity and computing boost the B5G-IIoT applications' performance both in terms of latency and reliability. The data analytic, storage, caching, and computing are yet the open research directions in B5G-IIoT. Open research problems also include implementing and optimizing self-organization, efficiency, and run-time features of cloud-based learning. The research also focuses on machine learning algorithms with configurable parameters and platforms that will aid decision-making in B5G-IIoT.

\section{Industrial Automation}

Manufacturing, supply chain management, energy management, and human resources are all industrial processes that heavily rely on OT and their expertise. Combining AI and B5G-IIoT will allow these operational processes to be enhanced and taken to a whole new level of precision. The integration of B5G-IIoT with AI, big spectrum data and data analytic enhance the services of B5G-IIoT. AI in B5G-IIoT connect the automotive industry, smart homes and machine tools to make things digital and more productive.

Everything in today's world is data-driven, whether industrial processes or smart home devices. Industrialists are currently unable to manage the massive amounts of data generated in an industrial complex or the B5G-IIoT ecosystem as a whole due to a lack of skilled human resources and reliable tools for productively utilizing big industrial data. AI is capable of autonomously managing itself and will be able to overcome the limitations of skilled human resources or tools, allowing for optimal utilization and optimization. A connected IIoT ecosystem of devices coupled with AI-powered analytical models can enhance not only the manufacturing operation, but the entire industrial process as well. The combination of AI and IIoT presents too many advantages to ignore or overlook in terms of dependability and reliability.

Automated mobile robotss (AMRs) are becoming more important for manufacturing companies to support their logistics operations. An AMR can move flexibly in a factory and can recognize and avoid obstacles at the highest safety level, and they can be programmed easily. Furthermore, the following two factors need to be addressed as efficiently as possible: (a) unpredictable delivery times and downtime, and (b) vulnerability to network attacks. The fierce competition in IIoT and market maturity give many legacy companies in infrastructure management a stronghold throughout the value chain by selling software. Take advantage of a hybrid cloud designed to offer manufacturers and plant operators a more open, flexible, and secure alternative for capturing and analyzing real-time data from IIoT [188]. The industrial sector must pass this transformational and evolutionary phase in order to survive, and only those who gain the most from the change will be able to survive.

\section{Spectral and Energy Efficient Designs}

Many stringent B5G-IIoT applications consume excessive energy and require high speed transmission as well. Thus, spectrum sharing and making energy efficient B5G-IIoT applications give new directions to the research community. Numerous methods to study the spectral and energy efficient designs have been done for WSN and some for IoT network, however a collective effort for the design of IIoT network is still an open problem.

An interesting research direction is the use of unmanned aerial vehicle (UAV) which will replace a large number of IIoT devices, particularly in agriculture, traffic, and monitoring, which will reduce power consumption and pollution. In the future, UAVs are expected to provide low-cost, high-efficiency IIoT systems. Another more useful and a very hot and promising technology for B5G-IIoT is transmission of sensor data to the mobile cloud. Green social networking (GSN) as a service can investigate the system, service, wireless network, and cloud management in terms of energy efficiency. Another direction is the use of M2M communication to reduce energy usage and hazardous emissions in automated IIoT systems. We need appropriate techniques for improving QoS parameters (bandwidth, delay, and throughput) in order to enhance the SE of B5G-IIoT system. Another interesting research direction is to develop a green IIoT, which will utilize less energy, search for new resources, and minimize the impact of the IoT on human health and the environment. Green IIoT poses an important contribution to achieving a smart, sustainable, and green environment. RF energy harvest is of critical importance for an energy-balanced communication in B5G-IIoT. It is necessary to conduct more research in order to design IIoT devices that can reduce $\mathrm{CO} 2$ emissions and energy consumption. Saving energy and reducing carbon dioxide emissions are critical to a green and sustainable life.

\section{E. Security and Privacy}

Security and privacy solutions for B5G-IIoT is always an open research topic and it is deemed as one of the major obstacle in the wide spread adoptability of this network. Owing to the increase in the inter-connectivity of the IIoT network, there is a need for a robust and secure transmission between the nodes. Standard IoT solutions can in general be applied to some of the applications of IIoT, however, some sophisticated techniques are required for applications with stringent safety concerns and resource constraint. Research in the direction of network security, data sharing and security, authentication and security monitoring for specific applications of IIoT is required.

In order to support industrial systems, which require realtime information, e.g., sensor data, and commands, security mechanisms should guarantee both security services and timely communication. Since most B5G-IIoT systems rely on large amounts of data, storing data in a third-party system is logical. Data confidentiality and the privacy of important assets should be the top concerns regarding the transfer of data to a third party. Several potential solutions to these problems have been identified, including homomorphic encryption and searchable encryption, but more research is needed with regard to scalability, the delivery of data from the cloud in time-critical applications, and reliable recovery mechanisms 
when third-party storage systems are compromised. Another important area for cryptographic research is the development of quantum-safe crypto-systems that scale with resourcesconstrained devices. A quantum-safe security scheme suitable for the IIoT is urgently needed, given the significance of the IIoT, and the benefits of public-key cryptography for managing key material for a large number of devices.

B5G-IIoT deployments often employ heterogeneous hardware and software solutions. This poses another research challenge. It is challenging to ensure that required security services are maintained as data spreads across multiple layers and an array of hardware and software. It is especially essential in situations where end-to-end encryption is required since data may travel across multiple hardware devices and software implementations, as well as across multiple vendors' hardware and software. Research into designing tamper-resistant hardware and maintaining operational safety in the face of such physical attacks are crucial for IIoT deployments in which an attacker may physically tamper with a device. It may be necessary to perform more research on the development of specific safety guarantees to be implemented with a complex IIoT system in safety-critical applications.

Another area of research is developing a privacy-preserving data collection and analytics approach. This can be accomplished by anonymizing data. Additionally, it may become necessary to verify the identities of devices by requesting that they self-identify without disclosing anything. Therefore, it is necessary to research and develop techniques for (pseudo) anonymization and zero-knowledge proofs that are suitable for the IIoT. The integration of the IIoT with other emerging technologies such as B5G-IIoT and blockchain is another important research direction.

\section{F. Machine/Deep Learning Solutions}

Many applications need real-time monitoring with closedloop controls in which the industry can monitor itself and adapt automatically to provide benefits. This gives rise to a new research domain of the integration of industry with machine learning solutions. Many stringent B5G-IIoT applications consume excessive energy. Thus, spectrum sharing and making energy-efficient B5G-IIoT applications give new directions to the research community.

In B5G-IIoT, source space and target space have the same properties. However, complex IIoT systems in reality will not only undergo a domain shift, but will also possess different characteristics. A heterogeneous domain consists of similar IIoT systems manufactured by different manufacturers and equipped with different sensors. In the field of heterogeneous unsupervised domain adaptation, especially for complex physical systems, there is little research, but it has considerable potential, especially for IIoT applications. A second approach is to use simulation environments and then adapt them to real-life applications. The data in the source domain will most likely be sufficient for this direction. An open research question is how to evaluate the plausibility of the generated samples in terms of physical system coherence. Furthermore, controlling data generation from a physical process perspective also is an open research question.
In IIoT applications with extensive and detailed simulation environments, reinforcement learning (RL) has been especially successful. In addition, the traditional applications of RL have been comparatively simple. Further research is needed to extend to more complex problems. At present, there is no consensus or consolidation on how physics-induced ML can be applied to industrial applications, despite several directions being pursued. This research needs to be developed and consolidated to improve the interpretability of the developed models and methods. Most studies, however, focused on vibration data that was preprocessed into image-like signals. An important question is if these approaches can be extended to complex data sets and time series. The plausibility of the generated samples and the effect of the generated faults on the accuracy of the algorithms needs to be evaluated further. In future studies, the composition and selection of the training datasets must also be addressed. It is necessary to decide continuously if new measured data should be incorporated into the training data and algorithms updated, or if the data is redundant or already contained in the dataset used for training algorithms. In addition, this becomes even more complex if the decision to include the new measurement data is made on a fleet level in IIoT systems rather than solely on an individual unit level. Furthermore, the algorithm is improved by selecting those observations that will lead to the closest improvement in performance.

\section{CONCLUSION}

IIoT has the potential to transform many aspects of our daily lives, including the environment, business, infrastructure, and lifestyle. This survey provides an in-depth assessment of IIoT, including the architecture and goals of the B5G-IIoT. The majority of the earlier literature focused on the architecture of IIoT. This primary focus of this paper is on the URLLC and eMBB techniques for 5G/B5G-IIoT applications. The tradeoff between URLLC and eMBB for 5G-IIoT is presented, and insights for various applications are discussed. Future wireless communication is expected to play a significant role in optimizing the reliability and throughput of IIoT.

\section{APPENDIX I: ACRONYMS}

$2 \mathrm{G} \quad$ second generation.

3G third generation.

3GPP third generation partnership project.

$4 \mathrm{G}$ forth generation.

$5 \mathrm{G} \quad$ fifth generation.

6G sixth generation.

6LoWPAN IPv6 over low-power wireless personal area networks.

AAH at-home healthcare.

AI artificial intelligence.

AMR automated mobile robots.

ANN artifical neural network. 


\begin{tabular}{|c|c|c|c|}
\hline AP & access point. & IIoT & industrial Internet of things. \\
\hline AR & augmented reality. & IoT & Internet of things. \\
\hline \multirow[t]{2}{*}{ AWGN } & additive white gaussian noise. & IP & internet protocol. \\
\hline & & IPV6 & internet protocol version 6 . \\
\hline B5G & beyond $5 \mathrm{G}$. & IT & information technology. \\
\hline $\mathrm{BCI}$ & bilateral computer interfaces. & ITU & Telecommunication \\
\hline $\mathrm{BDA}$ & big data analytic. & & Union. \\
\hline BLE & bluetooth low energy. & & \\
\hline \multirow[t]{2}{*}{$\mathrm{BS}$} & base station. & LoRa & low range. \\
\hline & & LoRaWAN & LoRa wide area network. \\
\hline $\mathrm{CA}$ & carrier aggregation. & LPD & low power devices. \\
\hline CAN & controller area network. & LPWA & low power wide area. \\
\hline CAPEX & capital expenditure. & LPWAN & low power wide area network. \\
\hline \multirow[t]{2}{*}{ CoMP } & coordinated multi-point transmission & LTE & long term evolution. \\
\hline & and reception. & LTE-M & long term evolution for machine. \\
\hline \multirow[t]{2}{*}{ CPMS } & cyber-physical manufacturing sys- & & \\
\hline & tems. & $\mathrm{M} 2 \mathrm{M}$ & machine to machine. \\
\hline CPS & cyber-physical systems. & MAC & medium access control. \\
\hline CSI & channel state information. & $\begin{array}{l}\text { MES } \\
\text { MIMO }\end{array}$ & $\begin{array}{l}\text { manufacturing execution system. } \\
\text { multiple-input multiple-output. }\end{array}$ \\
\hline $\mathrm{D} 2 \mathrm{D}$ & device to device. & mIoT & massive IoT. \\
\hline $\mathrm{DC}$ & dual connectivity. & ML & machine learning. \\
\hline DL & downlink. & mm-Wave & millimeter wave. \\
\hline DMRS & demodulation reference signals. & mMTC & massive MTC. \\
\hline DNS & domain name system. & MTC & machine type communication. \\
\hline DSS & dynamic spectrum sharing. & Multi-TRP & $\begin{array}{l}\text { multiple transmission and reception } \\
\text { architecture. }\end{array}$ \\
\hline \multirow[t]{2}{*}{ EASDF } & edge application server discovery & & \\
\hline & function. & NAN & neighbourhood area network. \\
\hline $\mathrm{EE}$ & energy efficiency. & NB-IoT & narrowband Internet of things. \\
\hline eMBB & enhanced mobile broadband. & $\mathrm{NFC}$ & near field communication. \\
\hline EPS & evolved packet system. & $\mathrm{NPN}$ & non-public networks. \\
\hline \multirow[t]{2}{*}{ EtherCAT } & ethernet for control automation tech- & NR & new radio. \\
\hline & nology. & NSA & non-standalone. \\
\hline FD & flexible data-rate. & OEM & original equipment manufacturer. \\
\hline \multirow[t]{2}{*}{ FDD } & frequency division duplex. & OPEX & operating expenditures. \\
\hline & & OT & operational technology. \\
\hline gNB & next generation nodeB. & & \\
\hline GNSS & global navigation satellite system. & $\mathrm{P} 2 \mathrm{P}$ & peer to peer. \\
\hline GPRS & general packet radio service. & $\mathrm{PDCCH}$ & physical downlink control channel. \\
\hline GPS & global positioning system. & PDSCH & physical downlink shared channel. \\
\hline \multirow[t]{2}{*}{ GSM } & global system for mobile communi- & PDU & protocol data unit. \\
\hline & cations. & PHY & physical layer. \\
\hline \multirow[t]{2}{*}{ GSN } & green social networking. & PoS & point of sale. \\
\hline & & ProfiBus & process field bus. \\
\hline HARQ & hybrid automatic repeat request. & ProfiNet & process field net. \\
\hline HART & highway addressable remote trans- & PSM & power saving mode. \\
\hline & ducer. & $\mathrm{PUCCH}$ & physical uplink control channel. \\
\hline HD & high definition. & PUSCH & physical uplink shared channel. \\
\hline IAB & integrated access and backhaul. & QoS & quality of service. \\
\hline
\end{tabular}


RAN radio access network.

RAT radio access technology.

RF radio frequency.

RFID radio-frequency identification.

RL reinforcement learning.

RRC radio resource control.

RTT round trip time.

SA standalone

SCADA supervisory control and data acquisition.

SDN software-defined-networking.

SE spectral efficiency.

SERCOS serial real-time communication system.

SNR signal to noise ratio.

TCP transmission control protocol.

TDD time division duplex.

TSC time-sensitive communications.

TSN time-sensitive networking.

TTI transmission time interval.

UAV unmanned aerial vehicle.

UDN ultra dense small cell network.

UE user equipment.

UL uplink.

UNB ultra narrow band.

URLLC ultra-reliable low latency communication.

URSP user-equipment route-selection policy.

V2I

$\mathrm{V} 2 \mathrm{~N}$

$\mathrm{V} 2 \mathrm{~V}$

$\mathrm{V} 2 \mathrm{X}$

VR

Wi-SUN wireless smart ubiquitous network.

WiFi

WLAN

WNAN

WPAN

WSN

WWAN

vehicle-to-infrastructure.

vehicle-to-network

vehicle-to-vehicle.

vehicle-to-everything.

virtual reality.

wireless fidelity.

wireless local area network.

wireless neighbourhood area network.

ZigBEE zonal intercommunication globalstandard.

\section{REFERENCES}

[1] Y. Iraqi and A. Al-Dweik, "Efficient information transmission using smart OFDM for IoT applications," IEEE Internet Things J., vol. 7, no. 9, pp. 8397-8409, Sept. 2020.

[2] H. Yahya, A. Al-Dweik, and E. Alsusa, "Power-tolerant NOMA using data-aware adaptive power assignment for IoT systems," IEEE Internet Things J., vol. 8, no. 19, pp. 14 896-14907, Oct. 2021.

[3] H. Yahya, A. Al-Dweik, Y. Iraqi, E. Alsusa, and a. ahmed, "A power and spectrum efficient uplink transmission scheme for QoS-constrained IoT networks," Sep 2021.

[4] S. Zafar, R. Hussain, F. Hussain, and S. Jangsher, "Interplay between big spectrum data and mobile internet of things: Current solutions and future challenges," Comput. Netw., vol. 163, p. 106879, Nov. 2019.

[5] C. Wu, D. Gunatilaka, M. Sha, and C. Lu, "Real-time wireless routing for industrial internet of things," in IEEE/ACM Intl. Conf. Internet Things Des. Implementation (IoTDI), Apr. 2018, pp. 261-266.

[6] M. Mohammadi, A. Al-Fuqaha, S. Sorour, and M. Guizani, "Deep learning for IoT big data and streaming analytics: A survey," IEEE Commun. Surveys Tuts., vol. 20, no. 4, pp. 2923-2960, June 2018.

[7] P. O'Donovan, K. Leahy, K. Bruton, and D. O'Sullivan, “An industrial big data pipeline for data-driven analytics maintenance applications in large-scale smart manufacturing facilities," J. Big Data, vol. 2, no. 25, pp. 1-26, Nov. 2015.

[8] A.-R. Sadeghi, C. Wachsmann, and M. Waidner, "Security and privacy challenges in industrial internet of things," in ACM/EDAC/IEEE Des. Autom. Conf. (DAC), June 2015, pp. 1-6.

[9] M. Amjad, A. Ahmed, M. Naeem, M. Awais, W. Ejaz, and A. Anpalagan, "Resource management in energy harvesting cooperative IoT network under QoS constraints," Sensors, vol. 18, no. 10, Oct. 2018.

[10] M. Awais, A. Ahmed, S. A. Ali, M. Naeem, W. Ejaz, and A. Anpalagan, "Resource management in multicloud IoT radio access network," IEEE Internet Things J., vol. 6, no. 2, pp. 3014-3023, Apr. 2019.

[11] A. Ahmed, M. Naeem, and A. Al-Dweik, "Joint optimization of sensors association and UAVs placement in IoT applications with practical network constraints," IEEE Access, vol. 9, pp. 7674-7689, Jan. 2021.

[12] M. Ahmad et al., "Device-centric communication in IoT: An energy efficiency perspective,” Trans. Emerging Telecommun. Technol., vol. 31, no. 2 , p. e3750, Oct. 2020.

[13] A. Ahmed, M. Awais, T. Akram, S. Kulac, M. Alhussein, and K. Aurangzeb, "Joint placement and device association of UAV base stations in IoT networks," Sensors, vol. 19, no. 9, Apr. 2019.

[14] M. Ali et al., "Devices to devices (Ds2Ds) communication: Towards energy efficient IoT," in IEEE Global Commun. Conf. (GLOBECOM), 2018, pp. 1-6.

[15] A. Al-Dweik, R. Muresan, M. Mayhew, and M. Lieberman, "IoTbased multifunctional scalable real-time enhanced road side unit for intelligent transportation systems," in IEEE Canadian Conf. Elect. Comput. Eng. (CCECE), 2017, pp. 1-6.

[16] M. A. Al-Jarrah, M. A. Yaseen, A. Al-Dweik, O. A. Dobre, and E. Alsusa, "Decision fusion for IoT-based wireless sensor networks," IEEE Internet Things J., vol. 7, no. 2, pp. 1313-1326, Feb. 2020.

[17] M. A. Al-Jarrah, M. A. Yaseen, and A. Al-Dweik, "Decision fusion for clustered WSNs over IoT infrastructure using 1-bit quantization," in Intl. Conf. Elect. Comput. Technol. Appl. (ICECTA), 2019, pp. 1-5.

[18] S. Zafar, S. Jangsher, O. Bouachir, M. Aloqaily, and J. Ben Othman, "QoS enhancement with deep learning-based interference prediction in mobile IoT," Comput. Commun., vol. 148, pp. 86-97, Dec. 2019.

[19] J. Cheng, W. Chen, F. Tao, and C.-L. Lin, "Industrial IoT in 5G environment towards smart manufacturing," J. Ind. Inf. Integr., vol. 10, pp. 10-19, June 2018.

[20] M. Bennis, M. Debbah, and H. V. Poor, "Ultrareliable and low-latency wireless communication: Tail, risk, and scale," Proc. IEEE, vol. 106, no. 10, pp. 1834-1853, Oct. 2018.

[21] L. Chettri and R. Bera, "A comprehensive survey on internet of things (IoT) toward 5G wireless systems," IEEE Internet Things J., vol. 7, no. 1 , pp. 16-32, Oct. 2020.

[22] J. Lin, W. Yu, N. Zhang, X. Yang, H. Zhang, and W. Zhao, "A survey on internet of things: Architecture, enabling technologies, security and privacy, and applications," IEEE Internet Things J., vol. 4, no. 5, pp. 1125-1142, Mar. 2017.

[23] M. Agiwal, A. Roy, and N. Saxena, "Next generation 5G wireless networks: A comprehensive survey," IEEE Commun. Surveys Tuts., vol. 18, no. 3, pp. 1617-1655, Feb. 2016. 
[24] M. R. Palattella et al., "Internet of things in the 5G era: Enablers, architecture, and business models," IEEE J. Sel. Areas Commun., vol. 34, no. 3, pp. 510-527, Mar. 2016.

[25] M. Shafi et al., "5G: A tutorial overview of standards, trials, challenges, deployment, and practice," IEEE J. Sel. Areas Commun., vol. 35, no. 6, pp. 1201-1221, June 2017.

[26] G. A. Akpakwu, B. J. Silva, G. P. Hancke, and A. M. Abu-Mahfouz, "A survey on $5 \mathrm{G}$ networks for the internet of things: Communication technologies and challenges," IEEE Access, vol. 6, pp. 3619-3647, Dec. 2018.

[27] F. Ghavimi and H.-H. Chen, "M2M communications in 3GPP LTE/LTE-A networks: Architectures, service requirements, challenges, and applications," IEEE Commun. Surveys Tuts., vol. 17, no. 2, pp. 525-549, 2015.

[28] H.-J. Kwon et al., "Licensed-assisted access to unlicensed spectrum in LTE release 13," IEEE Commun. Mag., vol. 55, no. 2, pp. 201-207, Feb. 2017.

[29] E. Pei and J. Jiang, "Performance analysis of licensed-assisted access to unlicensed spectrum in LTE release 13," IEEE Trans. Veh. Technol., vol. 68, no. 2, pp. 1446-1458, Feb. 2019.

[30] J. Andreoli-Fang and J. T. Chapman, "Latency reduction for mobile backhaul by pipelining LTE and DOCSIS," in IEEE Global Commun. Conf., 2017, pp. 1-6.

[31] P. Popovski, K. F. Trillingsgaard, O. Simeone, and G. Durisi, "5G wireless network slicing for eMBB, URLLC, and mMTC: A communication-theoretic view," IEEE Access, vol. 6, pp. 55765 55779 , Sept. 2018.

[32] C. I. Nwakanma, A. P. Anantha, F. B. Islam, J.-M. Lee, and D.-S. Kim, "3GPP release-16 for industrial internet of things and mission critical communications," in Intl. Conf. Informat. and Commun. Technol. Convergence (ICTC), 2020, pp. 403-406.

[33] W.-P. Nwadiugwu, C. I. Nwakanma, J.-M. Lee, and D.-S. Kim, "Achieving reliable URLLC-based network in industrial and military embedded systems," in Intl. Conf. Informat. and Commun. Technol. Convergence (ICTC), 2019, pp. 642-645.

[34] A. Anand, G. de Veciana, and S. Shakkottai, "Joint scheduling of URLLC and eMBB traffic in $5 \mathrm{G}$ wireless networks," IEEE/ACM Trans. Netw., vol. 28, no. 2, pp. 477-490, Feb. 2020.

[35] M. J. P. A. T. Amin Shahraki, Mahmoud Abbasi, "A comprehensive survey on 6G networks: Applications, core services, enabling technologies, and future challenges," IEEE Trans. Netw. Service Manag., June 2021.

[36] M. Alsharif, A. H. Kelechi, M. Albreem, S. A. Chaudhry, M. S. Zia, and S. Kim, "Sixth generation $(6 \mathrm{G})$ wireless networks: Vision, research activities, challenges and potential solutions," Symmetry, vol. 12, p. 676, April 2020

[37] X. Yang, Z. Zho, and B. Huang, "URLLC key technologies and standardization for $6 \mathrm{G}$ power internet of things," IEEE Commun. Standards Mag., vol. 5, no. 2, pp. 52-59, June 2021.

[38] J. Prados-Garzon, P. Ameigeiras, J. Ordonez-Lucena, P. Muñoz, O. Adamuz-Hinojosa, and D. Camps-Mur, "5G non-public networks: Standardization, architectures and challenges," IEEE Access, vol. 9, pp. 153 893-153 908, Nov. 2021.

[39] T. Panagiotis et al., "A cost-efficient 5G non-public network architectural approach: Key concepts and enablers, building blocks and potential use cases," Sensors, vol. 21, 082021.

[40] A. Mahmood et al., "Industrial IoT in 5G-and-beyond networks: Vision, architecture, and design trends," IEEE Trans. Ind. Informat., pp. 1-1, Sept. 2021.

[41] K. Mekki, E. Bajic, F. Chaxel, and F. Meyer, "A comparative study of LPWAN technologies for large-scale IoT deployment," ICT Express, vol. 5, no. 1, pp. 1-7, March 2019.

[42] T. O. Franck Muteba, Karim Djouani, "A comparative survey study on LPWA IoT technologies: Design, considerations, challenges and solutions," Procedia Comput. Sci., vol. 155, pp. 636-641, 2019.

[43] L. D. Xu, W. He, and S. Li, "Internet of things in industries: A survey," IEEE Trans. Ind. Informat., vol. 10, no. 4, pp. 2233-2243, Nov. 2014

[44] E. Sisinni, A. Saifullah, S. Han, U. Jennehag, and M. Gidlund, "Industrial internet of things: Challenges, opportunities, and directions," IEEE Trans. Ind. Informat., vol. 14, no. 11, pp. 4724-4734, Nov. 2018.

[45] S. Jaloudi, "Communication protocols of an industrial internet of things environment: A comparative study," Future Internet, vol. 11, no. 3, Mar. 2019

[46] P. P. Ray, "Creating values out of internet of things: An industrial perspective," J. Computer Netw. Commun., Oct. 2016.
[47] M. A. Siddiqi, H. Yu, and J. Joung, "5G ultra-reliable low-latency communication implementation challenges and operational issues with IoT devices," Electron., vol. 8, no. 9, Sept. 2019.

[48] W. Mao, Z. Zhao, Z. Chang, G. Min, and W. Gao, "Energy-efficient industrial internet of things: Overview and open issues," IEEE Trans. Ind. Informat., vol. 17, no. 11, pp. 7225-7237, Nov. 2021.

[49] N. Al-Falahy and O. Y. Alani, "Technologies for 5G networks: Challenges and opportunities," IT Professional, vol. 19, no. 1, pp. 12-20, Feb. 2017.

[50] E. Hossain, M. Rasti, H. Tabassum, and A. Abdelnasser, "Evolution toward 5G multi-tier cellular wireless networks: An interference management perspective," IEEE Wireless Commun., vol. 21, no. 3, pp. 118127, Jan. 2014.

[51] H. Ji, S. Park, J. Yeo, Y. Kim, J. Lee, and B. Shim, "Ultra-reliable and low-latency communications in 5G downlink: Physical layer aspects," IEEE Wireless Commun., vol. 25, no. 3, pp. 124-130, June 2018.

[52] C. Paniagua and J. Delsing, "Industrial frameworks for internet of things: A survey," IEEE Syst. J., vol. 15, no. 1, pp. 1149-1159, Mar 2021 .

[53] H. Wang and A. O. Fapojuwo, "A survey of enabling technologies of low power and long range machine-to-machine communications," IEEE Commun. Surveys Tuts., vol. 19, no. 4, pp. 2621-2639, 2017.

[54] K. P. Dirgantoro, W.-P. Nwadiugwu, J. M. Lee, and D.-S. Kim, "Dua fieldbus industrial IoT networks using edge server architecture," Manuf. Lett., vol. 24, pp. 108-112, Apr. 2020.

[55] Q. Yu and M. Gu, "Adaptive group routing and scheduling in multicast time-sensitive networks," IEEE Access, vol. 8, pp. 37855-37865, Feb. 2020.

[56] "IIoT: The current state of industrial connectivity and new technologies," https://omdia.tech.informa.com/OM004616/IIoTThe-current-state-of-industrial-connectivity-and-new-technologies

[57] R. Hassan, F. Qamar, M. K. Hasan, A. H. M. Aman, and A. S. Ahmed, "Internet of things and its applications: A comprehensive survey," Symmetry, vol. 12, no. 10, Oct. 2020

[58] R. Sanchez-Iborra and M.-D. Cano, "State of the art in LP-WAN solutions for industrial IoT services," Sensors, vol. 6, p. 5, May 2016

[59] M. S. Mahmoud and A. Mohamad, "A study of efficient power consumption wireless communication techniques/ modules for internet of things (IoT) applications," Advances Internet Things, vol. 6, pp. 19-29, April 2016.

[60] P. Chang, "Low power wide area networks, NB-IoT and the internet of things," http://docplayer.net/41844450-Low-power-wide-areanetworks-nb-iot-and-the-internet-of-things.html.

[61] B. Foubert and N. Mitton, "Long-range wireless radio technologies: A survey," Future Internet, vol. 12, no. 1, Jan. 2020.

[62] F. Foukalas, P. Pop, F. Theoleyre, C. A. Boano, and C. Buratti, "Dependable wireless industrial IoT networks: Recent advances and open challenges," in IEEE European Test Symp. (ETS), May 2019, pp. $1-10$.

[63] A. Sari, A. Lekidis, and I. Butun, Industrial Networks and IIoT: Now and Future Trends, 2020, pp. 3-55.

[64] S. Jo, J. Lee, J. Han, and S. Ghose, "P2P computing for intelligence of things," Peer-to-Peer Netw. Appl., vol. 13, Feb. 2020.

[65] S. Alkhalaf, "A control-driven autonomous authentication scheme for peer-to-peer control systems assisted industrial internet of things," Soft Comput., May 2021

[66] H. Muthukrishnan, B. Sunita, S. Najeerabanu, and V. Yasuvanth, "Observational study of WPAN and LPWA technologies for various IoT devices and its applications," Intl. J. Advanced Sci. Technol., vol. 29, pp. 4231 - 4243, May 2020.

[67] Y. Liu, M. Kashef, K. B. Lee, L. Benmohamed, and R. Candell, "Wireless network design for emerging IIoT applications: Reference framework and use cases," Proc. IEEE, vol. 107, no. 6, pp. 1166-1192, April 2019.

[68] P. Grossetete, "Demystifying 5G in industrial IoT," CISCO, Tech Rep., Feb. 2019. [Online]. Available: https://blogs.cisco.com/internetof-things/what-does-5g-look-like-for-industrial-iot

[69] M. Attaran, "The impact of 5G on the evolution of intelligent automation and industry digitization," J. ambient intell. humanized comput., Feb. 2021.

[70] N. Abosata, S. Al-Rubaye, G. Inalhan, and C. Emmanouilidis, "Internet of things for system integrity: A comprehensive survey on security, attacks and countermeasures for industrial applications," Sensors, vol. 21, no. 11, May 2021

[71] I. Ungurean and N. C. Gaitan, "A software architecture for the industrial internet of things-a conceptual model," Sensors, vol. 20, no. 19 , Sep. 2020 
[72] Z. Dawy, W. Saad, A. Ghosh, J. G. Andrews, and E. Yaacoub, "Toward massive machine type cellular communications," IEEE Wireless Commun., vol. 24, no. 1, pp. 120-128, Feb. 2017.

[73] R. L. G. Cavalcante, S. Stanczak, M. Schubert, A. Eisenblaetter, and U. Tuerke, "Toward energy-efficient $5 \mathrm{G}$ wireless communications technologies: Tools for decoupling the scaling of networks from the growth of operating power," IEEE Signal Process. Mag., vol. 31, no. 6, pp. 24-34, Nov. 2014

[74] M. N. Tehrani, M. Uysal, and H. Yanikomeroglu, "Device-to-device communication in $5 \mathrm{G}$ cellular networks: Challenges, solutions, and future directions," IEEE Commun. Mag., vol. 52, no. 5, pp. 86-92, May 2014.

[75] J. Kim, J. Lee, J. Kim, and J. Yun, "M2M service platforms: Survey, issues, and enabling technologies," IEEE Commun. Surveys Tuts., vol. 16, no. 1, pp. 61-76, 2014.

[76] D. Solomitckii, M. Gapeyenko, V. Semkin, S. Andreev, and Y. Koucheryavy, "Technologies for efficient amateur drone detection in 5G millimeter-wave cellular infrastructure," IEEE Commun. Mag., vol. 56, no. 1 , pp. 43-50, Jan. 2018

[77] C. Sexton, Q. Bodinier, A. Farhang, N. Marchetti, F. Bader, and L. A. DaSilva, "Enabling asynchronous machine-type D2D communication using multiple waveforms in 5G," IEEE Internet Things J., vol. 5, no. 2, pp. 1307-1322, April 2018.

[78] J. Wang, J. Liu, and N. Kato, "Networking and communications in autonomous driving: A survey," IEEE Commun. Surveys Tuts., vol. 21, no. 2, pp. 1243-1274, 2019.

[79] Z. Amjad, A. Sikora, B. Hilt, and J.-P. Lauffenburger, "Low latency V2X applications and network requirements: Performance evaluation," in IEEE Intell. Veh. Symp. (IV), June 2018, pp. 220-225.

[80] S. Husain, A. Kunz, A. Prasad, E. Pateromichelakis, K. Samdanis, and J. Song, "The road to 5G V2X: Ultra-high reliable communications," in IEEE Conf. Standards Commun. Netw. (CSCN), Oct. 2018, pp. 1-6.

[81] J. Lianghai, A. Weinand, B. Han, and H. D. Schotten, "Multi-RATs support to improve V2X communication," in IEEE Wireless Commun. Netw. Conf. (WCNC), Apr. 2018, pp. 1-6.

[82] J. Contreras-Castillo, S. Zeadally, and J. A. Guerrero-Ibañez, "Internet of vehicles: Architecture, protocols, and security," IEEE Internet Things J., vol. 5, no. 5, pp. 3701-3709, Oct. 2018.

[83] A. Kanavos, D. Fragkos, and A. Kaloxylos, "V2X communication over cellular networks: Capabilities and challenges," Telecom, vol. 2, no. 1 , pp. 1-26, Jan. 2021.

[84] D. Garcia-Roger, E. E. Gonzalez, D. Martin-Sacristan, and J. F Monserrat, "V2X support in 3GPP specifications: From 4G to 5G and beyond," IEEE Access, vol. 8, pp. 190 946-190 963, Oct. 2020.

[85] H. Bagheri et al., "5G NR-V2X: Toward connected and cooperative autonomous driving," IEEE Commun. Standards Mag., vol. 5, no. 1 , pp. 48-54, March 2021

[86] M. N. Ahangar, Q. Z. Ahmed, F. A. Khan, and M. Hafeez, "A survey of autonomous vehicles: Enabling communication technologies and challenges," Sensors, vol. 21, no. 3, Jan. 2021.

[87] X. Ge, "Ultra-reliable low-latency communications in autonomous vehicular networks," IEEE Trans. Veh. Technol., vol. 68, no. 5, pp. 5005-5016, May 2019.

[88] S. Chen et al., "Vehicle-to-everything (V2X) services supported by LTE-based systems and 5G," IEEE Commun. Standards Mag., vol. 1, no. 2, pp. 70-76, July 2017

[89] R. Molina-Masegosa and J. Gozalvez, "LTE-V for sidelink 5G V2X vehicular communications: A new 5G technology for short-range vehicleto-everything communications," IEEE Veh. Technol. Mag., vol. 12, no. 4, pp. 30-39, Dec. 2017.

[90] M. Fallgren et al., "Fifth-generation technologies for the connected car: Capable systems for vehicle-to-anything communications," IEEE Veh. Technol.y Mag., vol. 13, no. 3, pp. 28-38, Sept. 2018.

[91] N. H. Mahmood, M. Lopez, D. Laselva, K. Pedersen, and G. Berardinelli, "Reliability oriented dual connectivity for URLLC services in 5G new radio," in Intl. Symp. Wireless Commun. Syst. (ISWCS), Aug. 2018, pp. 1-6.

[92] D. Moltchanov, A. Ometov, S. Andreev, and Y. Koucheryavy, "Upper bound on capacity of $5 \mathrm{G}$ mmWave cellular with multi-connectivity capabilities," Electron. Lett., vol. 54, pp. 724-726(2), May 2018.

[93] V. Petrov et al., "Dynamic multi-connectivity performance in ultradense urban mmWave deployments," IEEE J. Sel. Areas Commun., vol. 35, no. 9, pp. 2038-2055, June 2017.

[94] _ _ "Achieving end-to-end reliability of mission-critical traffic in softwarized 5G networks," IEEE J. Sel. Areas Commun., vol. 36, no. 3 , pp. 485-501, Mar. 2018
[95] X. Ba, Y. Wang, H. Hai, Y. Chen, and Z. Liu, "Performance comparison of multi-connectivity with CoMP in $5 \mathrm{G}$ ultra-dense network," in IEEE Veh. Technol. Conf. (VTC Spring), June 2018, pp. 1-5.

[96] C. She, Z. Chen, C. Yang, T. Q. S. Quek, Y. Li, and B. Vucetic, "Improving network availability of ultra-reliable and low-latency communications with multi-connectivity," IEEE Trans. Commun., vol. 66, no. 11, pp. 5482-5496, Nov. 2018.

[97] T. Hobler, M. Simsek, and G. P. Fettweis, "Mission reliability for URLLC in wireless networks," IEEE Commun. Lett., vol. 22, no. 11, pp. 2350-2353, Nov. 2018.

[98] 3GPP, "Technical specification group radio access network, nr (release 15)," 3rd Gener. Partnership Project (3GPP), Tech. Rep., 2017. [Online]. Available: http://www.3gpp.org/ftp//Specs/archive/38

[99] C. Wang, Y. Chen, Y. Wu, and L. Zhang, "Performance evaluation of grant-free transmission for uplink URLLC services," in IEEE Veh. Technol. Conf. (VTC Spring), June 2017, pp. 1-6.

[100] T. Jacobsen et al., "System level analysis of uplink grant-free transmission for URLLC," in IEEE Globecom Workshops (GC Wkshps), Dec. 2017, pp. 1-6.

[101] Z. Zhou, R. Ratasuk, N. Mangalvedhe, and A. Ghosh, "Resource allocation for uplink grant-free ultra-reliable and low latency communications," in IEEE Veh. Technol. Conf. (VTC Spring), June 2018, pp. $1-5$.

[102] R. Abreu, T. Jacobsen, G. Berardinelli, K. Pedersen, I. Z. Kovács, and P. Mogensen, "Power control optimization for uplink grant-free URLLC," in IEEE Wireless Commun. Netw. Conf. (WCNC), Apr. 2018 , pp. 1-6.

[103] G. Berardinelli et al., "Reliability analysis of uplink grant-free transmission over shared resources," IEEE Access, vol. 6, pp. 23 602-23 611, Apr. 2018.

[104] A.-S. Bana, K. F. Trillingsgaard, P. Popovski, and E. de Carvalho, "Short packet structure for ultra-reliable machine-type communication: Tradeoff between detection and decoding," in IEEE Intl. Conf. Acoustics, Speech Signal Process. (ICASSP), April 2018, pp. 6608-6612.

[105] M. Iwabuchi et al., "5G field experimental trials on URLLC using new frame structure," in IEEE Globecom Workshops (GC Wkshps), Dec. 2017, pp. 1-6.

[106] S.-Y. Lien, S.-L. Shieh, Y. Huang, B. Su, Y.-L. Hsu, and H.-Y. Wei, " $5 \mathrm{G}$ new radio: Waveform, frame structure, multiple access, and initia access," IEEE Commun. Mag., vol. 55, no. 6, pp. 64-71, June 2017.

[107] A. Ijaz et al., "Enabling massive IoT in 5G and beyond systems: PHY radio frame design considerations," IEEE Access, vol. 4, pp. 33223339, June 2016.

[108] E. Dahlman and S. Parkvall, "NR - the new 5G radio-access technology," in IEEE Veh. Technol. Conf. (VTC Spring), June 2018, pp. 1-6.

[109] S. Parkvall, E. Dahlman, A. Furuskar, and M. Frenne, "NR: The new 5G radio access technology," IEEE Commun. Standards Mag., vol. 1, no. 4, pp. 24-30, Dec. 2017.

[110] Z. E. Ankarali, B. Pekoz, and H. Arslan, "Flexible radio access beyond 5G: A future projection on waveform, numerology, and frame design principles," IEEE Access, vol. 5, pp. 18 295-18 309, Mar. 2017.

[111] B. Wang, L. Dai, Z. Wang, N. Ge, and S. Zhou, "Spectrum and energyefficient beamspace MIMO-NOMA for millimeter-wave communications using lens antenna array," IEEE J. Sel. Areas Commun., vol. 35, no. 10, pp. 2370-2382, July 2017.

[112] Y. Sun, D. W. K. Ng, Z. Ding, and R. Schober, "Optimal joint power and subcarrier allocation for full-duplex multicarrier non-orthogonal multiple access systems," IEEE Trans. Commun., vol. 65, no. 3, pp. 1077-1091, Mar. 2017.

[113] Z. Wei, D. W. K. Ng, J. Yuan, and H.-M. Wang, "Optimal resource allocation for power-efficient MC-NOMA with imperfect channel state information," IEEE Trans. Commun., vol. 65, no. 9, pp. 3944-3961, Sep. 2017.

[114] A. Akbar, S. Jangsher, and F. A. Bhatti, "Noma and 5G emerging technologies: A survey on issues and solution techniques," Computer Networks, vol. 190, p. 107950, May 2021.

[115] Q. Sun, S. Han, C.-L. I, and Z. Pan, "On the ergodic capacity of MIMO NOMA systems," IEEE Wireless Commun. Lett., vol. 4, no. 4, pp. 405-408, Aug. 2015.

[116] H. Zhang, N. Yang, K. Long, M. Pan, G. K. Karagiannidis, and V. C. M. Leung, "Secure communications in NOMA system: Subcarrier assignment and power allocation," IEEE J. Sel. Areas Commun., vol. 36, no. 7, pp. 1441-1452, July 2018.

[117] Q. Wu, W. Chen, D. W. K. Ng, and R. Schober, "Spectral and energyefficient wireless powered IoT networks: NOMA or TDMA?" IEEE Trans. Veh. Technol., vol. 67, no. 7, pp. 6663-6667, July 2018. 
[118] D. Bharadia, E. McMilin, and S. Katti, "Full duplex radios," $S I G-$ COMM Comput. Commun. Rev., vol. 43, no. 4, p. 375-386, Aug. 2013.

[119] T. Riihonen and R. Wichman, Full-Duplex Protocol Design for $5 G$ Networks, 2017, p. 172-187.

[120] A. Sabharwal, P. Schniter, D. Guo, D. W. Bliss, S. Rangarajan, and R. Wichman, "In-band full-duplex wireless: Challenges and opportunities," IEEE J. Sel. Areas Commun., vol. 32, no. 9, pp. 1637-1652, June 2014.

[121] Y. Sun, D. W. K. Ng, J. Zhu, and R. Schober, "Multi-objective optimization for robust power efficient and secure full-duplex wireless communication systems," IEEE Trans. Wireless Commun., vol. 15, no. 8, pp. 5511-5526, Apr. 2016.

[122] Z. Zhang, K. Long, A. V. Vasilakos, and L. Hanzo, "Full-duplex wireless communications: Challenges, solutions, and future research directions," Proc. IEEE, vol. 104, no. 7, pp. 1369-1409, July 2016.

[123] J. Sachs, G. Wikstrom, T. Dudda, R. Baldemair, and K. Kittichokechai, "5G radio network design for ultra-reliable low-latency communication," IEEE Netw., vol. 32, no. 2, pp. 24-31, Apr. 2018.

[124] 3GPP, "Study on new radio access technology physical layer aspects (release 14)," 3rd Gener. Partnership Project (3GPP), Tech. Rep. 36.331, 2017. [Online]. Available: https://portal.3gpp.org/desktopmodules/Specifications/ SpecificationDetails.aspx ?specificationId $=3066$

[125] A. Hazareena and B. A. Mustafa, "A survey: On the waveforms for 5G," in Intl. Conf. Electron., Commun. Aerosp. Technol. (ICECA), Mar. 2018, pp. 64-67.

[126] M. Renfors, J. Yli-Kaakinen, and M. Valkama, "Power amplifier effects on frequency localized 5G candidate waveforms," in European Wireless Conf., May 2016, pp. 1-5.

[127] M. Abdelaziz, L. Anttila, M. Renfors, and M. Valkama, "PAPR reduction and digital predistortion for non-contiguous waveforms with welllocalized spectrum," in Intl. Symp. Wireless Commun. Syst. (ISWCS), Sept. 2016, pp. 581-585.

[128] J. Yli-Kaakinen et al., "Efficient fast-convolution-based waveform processing for $5 \mathrm{G}$ physical layer," IEEE J. Sel. Areas Commun., vol. 35, no. 6, pp. 1309-1326, June 2017.

[129] R. Vannithamby and S. Talwar, New Physical-layer Waveforms for $5 G$, 2017, pp. 303-341.

[130] A. Karimi, K. I. Pedersen, N. H. Mahmood, J. Steiner, and P. Mogensen, "5G centralized multi-cell scheduling for URLLC: Algorithms and system-level performance," IEEE Access, vol. 6, pp. 72 253-72 262, Nov. 2018.

[131] M. Kamel, W. Hamouda, and A. Youssef, "Ultra-dense networks: A survey," IEEE Commun. Surveys Tuts., vol. 18, no. 4, pp. 2522-2545, May 2016.

[132] F. Al-Turjman, E. Ever, and H. Zahmatkesh, "Small cells in the forthcoming 5G/IoT: Traffic modelling and deployment overview," IEEE Commun. Surveys Tuts., vol. 21, no. 1, pp. 28-65, Aug. 2019.

[133] J. Rodriguez, Small Cells for 5G Mobile Networks, 2014, pp. 63-104.

[134] S. M. Ahsan Kazmi et al., "Resource management in dense heterogeneous networks," in 17th Asia-Pacific Netw. Operations Manage. Symp. (APNOMS), Aug. 2015, pp. 440-443.

[135] S. Han, C.-1. I, Z. Xu, and C. Rowell, "Large-scale antenna systems with hybrid analog and digital beamforming for millimeter wave $5 \mathrm{G}$," IEEE Commun. Mag., vol. 53, no. 1, pp. 186-194, Jan. 2015.

[136] M. Xiao et al., "Millimeter wave communications for future mobile networks," IEEE J. Sel. Areas Commun., vol. 35, no. 9, pp. 1909-1935, Sept. 2017

[137] S. A. Busari, K. M. S. Huq, S. Mumtaz, L. Dai, and J. Rodriguez, "Millimeter-wave massive MIMO communication for future wireless systems: A survey," IEEE Commun. Surveys Tuts., vol. 20, no. 2, pp. 836-869, Dec. 2018.

[138] X. Wang et al., "Millimeter wave communication: A comprehensive survey," IEEE Commun. Surveys Tuts., vol. 20, no. 3, pp. 1616-1653, June 2018.

[139] I. A. Hemadeh, K. Satyanarayana, M. El-Hajjar, and L. Hanzo, "Millimeter-wave communications: Physical channel models, design considerations, antenna constructions, and link-budget," IEEE Commun. Surveys Tuts., vol. 20, no. 2, pp. 870-913, Dec. 2018.

[140] D. Zhang, C. C. Chan, and G. Y. Zhou, "Enabling industrial internet of things (IIoT) towards an emerging smart energy system," Global Energy Interconnection, vol. 1, no. 1, pp. 39-47, Jan. 2018.

[141] J. Gubbi, R. Buyya, S. Marusic, and M. Palaniswami, "Internet of things (IoT): A vision, architectural elements, and future directions," Future Gener. Comput. Syst., vol. 29, no. 7, pp. 1645-1660, Sept. 2013.
[142] A. Ghosh, A. Maeder, M. Baker, and D. Chandramouli, "5G evolution: A view on $5 \mathrm{G}$ cellular technology beyond 3GPP release 15," IEEE Access, vol. 7, pp. $127639-127651$, Sept. 2019.

[143] C. I. Nwakanma, A. P. Anantha, F. B. Islam, J.-M. Lee, and D.-S. Kim, "3GPP release-16 for industrial internet of things and mission critical communications," in Intl. Conf. Informat. Commun. Technol. Convergence (ICTC), 2020, pp. 403-406.

[144] B. Lee and H. Yang, "Massive MIMO for industrial internet of things in cyber-physical systems," IEEE Trans. Ind. Informat., vol. 14, no. 6 , pp. 2641-2652, June 2018.

[145] J. Zong et al., "Smart user pairing for massive MIMO enabled industrial IoT communications," in IEEE Conf. Comput. Commun. Workshops (INFOCOM WKSHPS), 2020, pp. 207-212.

[146] Z. Shi, X. Xie, H. Lu, H. Yang, M. Kadoch, and M. Cheriet, "Deepreinforcement-learning-based spectrum resource management for industrial internet of things," IEEE Internet Things J., vol. 8, no. 5, pp. 3476-3489, Mar. 2021.

[147] M. Agiwal, H. Kwon, S. Park, and H. Jin, "A survey on 4G-5G dual connectivity: Road to 5G implementation," IEEE Access, vol. 9, pp. 16193-16210, Jan. 2021.

[148] W. Mao, Z. Zhao, Z. Chang, G. Min, and W. Gao, "Energy-efficient industrial internet of things: Overview and open issues," IEEE Trans. Ind. Informat., vol. 17, no. 11, pp. 7225-7237, Nov. 2021.

[149] D. Zhang, C. C. Chan, and G. Y. Zhou, "Enabling industrial internet of things (IIoT) towards an emerging smart energy system," Global Energy Interconnection, vol. 1, no. 1, pp. 39-47, 2018. [Online]. Available: https://www.sciencedirect.com/science/article/pii/ S2096511718300057

[150] D. C. Nguyen et al., "6G internet of things: A comprehensive survey," 2021.

[151] J. Yang et al., "Ultra-reliable communications for industrial internet of things: Design considerations and channel modeling," IEEE Netw. vol. 33, no. 4, pp. 104-111, Aug. 2019.

[152] E. J. Khatib and R. Barco, "Optimization of 5G networks for smart logistics," Energies, vol. 14, no. 6, Mar. 2021.

[153] P. Schulz et al., "Latency critical IoT applications in 5G: Perspective on the design of radio interface and network architecture," IEEE Commun. Mag., vol. 55, no. 2, pp. 70-78, Feb. 2017.

[154] S. M. A. A. Abir, A. Anwar, J. Choi, and A. S. M. Kayes, "IoT-enabled smart energy grid: Applications and challenges," IEEE Access, vol. 9 , pp. 50 961-50981, Mar. 2021

[155] Z. Davoody-Beni, N. Sheini-Shahvand, H. Shahinzadeh, M. Moazzami, M. Shaneh, and G. B. Gharehpetian, "Application of IoT in smart grid: Challenges and solutions," in Iranian Conf. Signal Process. Intell. Syst. (ICSPIS), Dec. 2019, pp. 1-8.

[156] S. A. Ashraf, I. Aktas, E. Eriksson, K. W. Helmersson, and J. Ansari, "Ultra-reliable and low-latency communication for wireless factory automation: From LTE to 5G," in IEEE Intl. Conf. Emerging Technol. Factory Autom. (ETFA), Sep. 2016, pp. 1-8.

[157] N. Brahmi, O. N. C. Yilmaz, K. W. Helmersson, S. A. Ashraf, and J. Torsner, "Deployment strategies for ultra-reliable and low-latency communication in factory automation," in IEEE Globecom Workshops (GC Wkshps), Dec. 2015, pp. 1-6.

[158] J. S. Walia, H. Hammainen, and H. Flinck, "Future scenarios and value network configurations for industrial 5G," in Intl. Conf. Netw. Future (NOF), Nov. 2017, pp. 79-84.

[159] H. A. Munz and J. Ansari, "An empirical study on using D2D relaying in 5G for factory automation," in IEEE Wireless Commun. Netw. Conf. Workshops (WCNCW), Apr. 2018, pp. 149-154.

[160] P. Rost et al., "Customized industrial networks: Network slicing trial at hamburg seaport," IEEE Wireless Commun., vol. 25, no. 5, pp. 48-55, Oct. 2018.

[161] V. Theodorou, K. V. Katsaros, A. Roos, E. Sakic, and V. Kulkarni, "Cross-domain network slicing for industrial applications," in European Conf. Netw. Commun. (EuCNC), June 2018, pp. 209-213.

[162] N. Ericsson, T. Lennvall, J. Akerberg, and M. Bjorkman, "Custom simulation of industrial wireless sensor and actuator network for improved efficiency during research and development," in IEEE Intl. Conf. Emerging Technol. Factory Autom. (ETFA), Sept. 2017, pp. 1-8.

[163] L. Liu and W. Yu, "A D2D-based protocol for ultra-reliable wireless communications for industrial automation," IEEE Trans. Wireless Commun., vol. 17, no. 8, pp. 5045-5058, Aug. 2018.

[164] C. Li et al., "5G-based systems design for tactile internet," Proc. IEEE, vol. 107, no. 2, pp. 307-324, Feb. 2019.

[165] F. Voigtlander, A. Ramadan, J. Eichinger, C. Lenz, D. Pensky, and A. Knoll, " $5 \mathrm{G}$ for robotics: Ultra-low latency control of distributed 
robotic systems," in Intl. Symp. Comput. Sci. Intell. Controls (ISCSIC), Oct. 2017, pp. 69-72.

[166] J. Ansari et al., "Demo: a realistic use-case for wireless industrial automation and control," in Intl. Conf. Netw. Syst. (NetSys), March 2017, pp. 1-2.

[167] A. Fellan, C. Schellenberger, M. Zimmermann, and H. D. Schotten, "Enabling communication technologies for automated unmanned vehicles in industry 4.0," in Intl. Conf. Inf. Commun. Technol. Convergence (ICTC), Nov. 2018, pp. 171-176.

[168] P. Duan, Y. Jia, L. Liang, J. Rodriguez, K. M. S. Huq, and G. Li, "Space-reserved cooperative caching in 5G heterogeneous networks for industrial IoT," IEEE Trans. Ind. Informat., vol. 14, no. 6, pp. 27152724, June 2018.

[169] L. Lyu, C. Chen, S. Zhu, and X. Guan, "5G enabled codesign of energyefficient transmission and estimation for industrial IoT systems," IEEE Trans. Ind. Informat., vol. 14, no. 6, pp. 2690-2704, June 2018.

[170] S. Hu et al., "Nonorthogonal interleave-grid multiple access scheme for industrial internet of things in 5G network," IEEE Trans. Ind. Informat., vol. 14, no. 12, pp. 5436-5446, Dec. 2018.

[171] T. Zheng, Y. Qin, H. Zhang, and S. Kuo, "Adaptive power control for mutual interference avoidance in industrial internet-of-things," China Commun., vol. 13, no. Supplement 1, pp. 124-131, Sept. 2016.

[172] E. E. Ugwuanyi, S. Ghosh, M. Iqbal, and T. Dagiuklas, "Reliable resource provisioning using bankers' deadlock avoidance algorithm in MEC for industrial IoT," IEEE Access, vol. 6, pp. 43327-43 335, Aug. 2018

[173] L. Lyu, C. Chen, S. Zhu, N. Cheng, B. Yang, and X. Guan, "Control performance aware cooperative transmission in multiloop wireless control systems for industrial IoT applications," IEEE Internet Things J., vol. 5, no. 5, pp. 3954-3966, Oct. 2018.

[174] U. Challita, K. Hiltunen, and M. Tercero, "Performance evaluation for the co-existence of eMBB and URLLC networks: Synchronized versus unsynchronized TDD," in IEEE Veh. Technol. Conf. (VTC2019-Fall), Sept. 2019, pp. 1-6.

[175] R. Kassab, O. Simeone, and P. Popovski, "Coexistence of URLLC and eMBB services in the C-RAN uplink: An information-theoretic study," in IEEE Global Commun. Conf. (GLOBECOM), Feb. 2018, pp. 1-6.

[176] M. Alsenwi, N. H. Tran, M. Bennis, A. Kumar Bairagi, and C. S Hong, "eMBB-URLLC resource slicing: A risk-sensitive approach," IEEE Commun. Lett., vol. 23, no. 4, pp. 740-743, April 2019.

[177] W.-R. Wu, P.-Y. Lin, and Y.-H. Lee, "An indicator-free eMBB and URLLC multiplexed scheme for 5G downlink system," in IEEE Veh. Technol. Conf. (VTC2019-Fall), Sept. 2019, pp. 1-5.

[178] A. K. Bairagi et al., "Coexistence mechanism between eMBB and uRLLC in 5G wireless networks," IEEE Trans. Commun., vol. 69, no. 3, pp. 1736-1749, March 2021.

[179] D. Qiao, M. C. Gursoy, and S. Velipasalar, "Throughput-delay tradeoffs with finite blocklength coding over multiple coherence blocks," IEEE Trans. Commun., vol. 67, no. 8, pp. 5892-5904, Aug. 2019.

[180] M. Gursoy, "Throughput analysis of buffer-constrained wireless systems in the finite blocklength regime," J. Wireless Commun. Netw. June 2013.

[181] W. Yang et al., "Narrowband wireless access for low-power massive internet of things: A bandwidth perspective," IEEE Wireless Commun., vol. 24, no. 3, pp. 138-145, June 2017.

[182] M. U. Baig, L. Yu, Z. Xiong, A. Høst-Madsen, H. Li, and W. Li, "On the energy-delay tradeoff in streaming data: Finite blocklength analysis," IEEE Trans. Inf. Theory, vol. 66, no. 3, pp. 1861-1881, Mar. 2020.

[183] D. B. Avancini, J. Rodrigues, R. Rabêlo, A. Das, S. Kozlov, and P. Solic, "A new iot-based smart energy meter for smart grids," Intl. J. Energy Research, vol. 1, pp. 1-14, Feb. 2020.

[184] Z. Pang, Q. Chen, J. Tian, L. Zheng, and E. Dubrova, "Ecosystem analysis in the design of open platform-based in-home healthcare terminals towards the internet-of-things," in Intl. Conf. Advanced Commun. Technol. (ICACT), Jan. 2013, pp. 529-534.

[185] M. C. Domingo, "An overview of the internet of things for people with disabilities," J. Netw. Comput. Appl., vol. 35, no. 2, pp. 584-596, Mar. 2012

[186] H. Alemdar and C. Ersoy, "Wireless sensor networks for healthcare: A survey," Comput. Netw., vol. 54, no. 15, pp. 2688-2710, Oct. 2010

[187] I. Plaza, L. Martin, S. Martin, and C. Medrano, "Mobile applications in an aging society: Status and trends," J. Syst. Softw., vol. 84, no. 11, pp. 1977-1988, Nov. 2011.

[188] L. Romeo, A. Petitti, R. Marani, and A. Milella, "Internet of robotic things in smart domains: Applications and challenges," Sensors, vol. 20, no. 12, May 2020

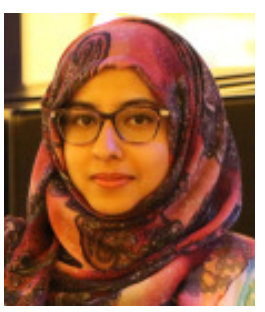

Benish Sharfeen Khan currently pursuing the Ph.D. degree with the Institute of Space Technology, Islamabad, Pakistan and her M.S. in electrical engineering (Wireless Communication) from Institute of Space Technology, Islamabad, Pakistan and B.S. degree in electronics engineering from International Islamic University Islamabad (IIUI), She is working as full time faculty in the department of electrical engineering at the National University of Modern Languages, Islamabad, Pakistan. Her research interest include cooperative communication, mobile relays, Internet of Things, 5G networks, small cells and machine learning.

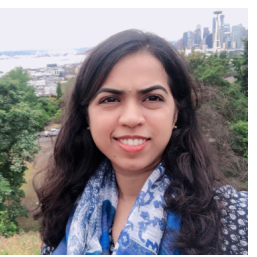

Sobia Jangsher received her B.E. degree in electronics engineering and M.S. in communication system engineering from National University of Science and Technology (NUST), Pakistan and PhD in Wireless Communication from The University of Hong Kong, Hong Kong. She is currently affiliated with the Department of Electrical Engineering and Computer Science, Khalifa University, Abu Dhabi, UAE She is also working as an Assistant Professor in Institute of Space Technology, Islamabad, Pakistan. Her research mainly focuses on resource allocation in future wireless communication systems.

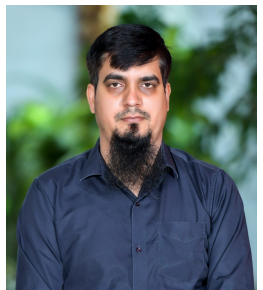

Ashfaq Ahmed received the M.S. and Ph.D. Degree from the Department of Electronics and Telecommunications, Politecnico di Torino, Torino, Italy, in 2010 and 2014, respectively. He is currently affiliated with the center for cyber-physical systems, Department of Electrical Engineering and Computer Science, Khalifa University, Abu Dhabi, UAE. He worked as an Assistant Professor at the Department of Electrical \& Computer Engineering, COMSATS University Islamabad, Wah campus, Pakistan from March 2014 to March 2021

His current research interests include the topics related to applied optimization, targeting telecommunication and smart system applications, ASICs and FPGA design and implementation of digital signal processing architectures, especially the areas closely related to digital communications, and video and image coding.

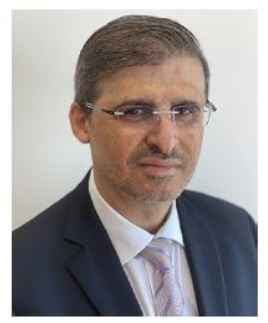

Arafat Al-Dweik (Senior Member, IEEE) received the B.Sc. degree in telecommunication engineering from Yarmouk University, Jordan, in 1994, and the M.S. (summa cum laude) and Ph.D. (magna cum laude) degrees in electrical engineering from Cleveland State University, Cleveland, OH, USA, in 1998 and 2001, respectively. He was with Efficient Channel Coding, Inc., Cleveland, from 1999 to 2001, where he was a Research and Development Engineer working on advanced modulation, coding, and synchronization techniques. From 2001 to 2003, he was the Head of the Department of Information Technology, Arab American University, Palestine. From 2003 to 2012, he was with the Communications Engineering Department, Khalifa University, UAE. From 2013 to 2014, he was an Associate Professor with the University of Guelph, Guelph, ON, Canada. He has been a Visiting Research Fellow with the School of Electrical, Electronic and Computer Engineering, Newcastle University, Newcastle upon Tyne, U.K., since 2006. He is also a Research Professor and a Member of the School of Graduate Studies, Western University, London, ON, Canada. He has received several research awards and he was a recipient of the Fulbright Scholarship from 1997 to 1999 . He was a TPC Member in several major conferences, such as IEEE GLOBECOM, ICC, PIMRC, and WCNC. He has extensive editorial experience where he serves as an Associate Editor for the IEEE TRANSACTIONS ON VEHICULAR TECHNOLOGY and IET Communications. 